\title{
A Defect-Correction Method for Time-Dependent Viscoelastic Fluid Flow Based on SUPG Formulation
}

\author{
Yunzhang Zhang, ${ }^{1,2}$ Yanren Hou, ${ }^{1}$ and Ganshan Yang ${ }^{3,4}$ \\ ${ }^{1}$ School of Science, Xi'an Jiaotong University, Xi'an 710049, China \\ ${ }^{2}$ School of Mathematics and Statistics, Henan University of Science and Technology, \\ Luoyang 471003, China \\ ${ }^{3}$ Institute of Mathematics Science, Yunnan Normal University, Kunming 650092, China \\ ${ }^{4}$ Department of Mathematics, Yunnan Nationalities University, Kunming 650031, China
}

Correspondence should be addressed to Ganshan Yang, ganshanyang@yahoo.com.cn

Received 22 November 2010; Revised 13 March 2011; Accepted 29 April 2011

Academic Editor: M. De la Sen

Copyright (c) 2011 Yunzhang Zhang et al. This is an open access article distributed under the Creative Commons Attribution License, which permits unrestricted use, distribution, and reproduction in any medium, provided the original work is properly cited.

\begin{abstract}
A defect-correction mixed finite element method for solving the time-dependent JohnsonSegalman viscoelastic equations in two dimensions is given. In the defect step, the constitutive equation is computed with the artificially reduced Weissenberg parameter for stability, and the resulting residual is corrected in the correction step on the same grid. A streamline upwind PetrovGalerkin (SUPG) approximation is used to stabilize the hyperbolic character of the constitutive equation for the stress. We establish a priori error estimates for the defect step and the first correction step of the defect correction method. The derived theoretical results are supported by numerical tests.
\end{abstract}

\section{Introduction}

Time-dependent calculations of viscoelastic flows are important to the understanding of many problems in non-Newtonian fluid mechanics, particularity those related to flow instabilities. Error analysis of finite element approximations to time-dependent viscoelastic flow was first analyzed by Baranger and Wardi in [1], using an implicit Euler temporal discretization and a discontinuous Galerkin (DG) approximation for the hyperbolic constitutive equation. In [2,3], Ervin and Miles have analyzed the problem using an implicit Euler time discretization and an SUPG discretization for the constitutive equation. The key of SUPG method, first introduced by Hughes and Brooks [4], is to stabilize the numerical solution by adding dissipative effects limited to the direction in which their influence 
is needed. Error analysis of a modified Euler-SUPG approximation to time-dependent viscoelastic flow problem was presented by Bensaada and Esselaoui in [5]. In [6], Ervin and Heuer have proposed a Crank-Nicolson time discretization scheme for this problem. A fractional step $\theta$-method for the time-dependent problem is described and analyzed in [7]. The reader may find more relevant work, for example, $[8,9]$ and the reference therein.

In addition, when the Weissenberg number increases, boundary layers for the stress develop. This will add to the difficulty of computing accurate numerical approximations. There are also many papers in developing stable numerical algorithms for high Weissenberg number flows (see [10-15] and the references therein) over the years. In [10-12], a defectcorrection method has successfully applied in steady viscoelastic and Oseen-viscoelastic fluid flow model, aiming at high Weissenberg number. The basic idea of defect correction method for viscoelastic fluid flow is as follows. In the defect step, the Weissenberg number is reduced artificially by using a mesh-dependent parameter to obtain better convergence of an iteration scheme. Then, in a correction step, the initial approximation is improved using the residual correction. The defect correction method is an iterative improvement technique for increasing the accuracy of a numerical solution without applying a grid refinement. Due to its good efficiency, there are many works devoted to this method, for example, [16-22].

Inspired by $[2,3,10-12,21,22]$, in this paper, we will use the defect correction method for solving the time-dependent Johnson-Segalman viscoelastic equations in two dimensions. Approximation in space is made by finite element (FE) method. Approximate stress, velocity, and pressure are, respectively, $P_{1}$ continuous, $P_{2}$ continuous, and $P_{1}$ continuous. As the constitutive equation has hyperbolic character for the stress, we use streamline upwind Petrov-Galerkin (SUPG) formulation to stabilize the constitutive equation. Approximate in time is made by Euler scheme. Finally, we establish a priori error estimates for the defect step and the first correction step of the defect correction method. While in [22], the authors present this problem by DG stabilized style, we know that these two styles have many differences. Choice of the Johnson and Segalman model is not essential, and the results obtained can be extended to more realistic differential models likes those of Phan-Thien and Tanner, Giesekus and Oldroyd, provided a Newtonian viscosity is present.

Detailed descriptions, analysis, and numerical examples of the defect correction method for the viscoelastic fluid flow are presented in the rest of the paper as follows. In the next section, we present the time-dependent viscoelastic fluid flow of the Johnson-Segalman model and its variational formulation. In Section 3, we introduce finite element spaces and present the fully discrete approximation scheme (Euler in time, FE in space). Defect correction algorithm is given in Section 4. Then a priori error estimates for the defect step and the first correction step of the defect correction method are derived in Section 5. Numerical results are presented in Section 6. Finally, a summary and discussion of continuing work are presented in Section 7.

\section{The Johnson-Segalman Model and Its Variational Formulation}

In this section, we consider a fluid flowing in a connected, bounded polygonal domain $\Omega \subset$ $\mathbb{R}^{d^{\prime}}, d^{\prime}=2$ with the Lipschitz continuous boundary $\Gamma$.

The following notation will be used. $R^{2}$ is equipped with cartesian coordinates $x_{i}, i=$ 1,2. For a function $u, \partial u / \partial x_{i}$ is written $u_{, i}$ and $\partial u / \partial t$ is written $u_{t}$. Einstein's convention of summation is used. For a scalar function $p$, gradient of $p$ is a vector $\nabla p$ with $(\nabla p)_{i}=p_{i}$. For a vector $\mathbf{u}$, gradient of $\mathbf{u}$ is a tensor $\nabla \mathbf{u}$ with $(\nabla \mathbf{u})_{i j}=\mathbf{u}_{i, j}$. For a vector function $\mathbf{u}$, divergence 
of $\mathbf{u}$ is a scalar $\nabla \mathbf{u}=\mathbf{u}_{i, i}$. with $(\nabla \mathbf{u})_{i j}=\mathbf{u}_{i, j}$. For a tensor function $\tau$ and a vector function $\mathbf{u}$, divergence of $\tau$ is a vector $\nabla \cdot \tau$ with $(\nabla \cdot \tau)_{i}=\tau_{i j, j}$ and $\mathbf{u} \cdot \nabla$ denotes the operator $u_{i}\left(\partial / \partial x_{i}\right)$.

Let $T>0$ be a given final time, we consider the time-dependent Johnson-Segalman viscoelastic equations as follows:

$$
\begin{aligned}
& \operatorname{Re}\left(\mathbf{u}_{t}+u \cdot \nabla u\right)-\nabla \cdot \sigma-2(1-\alpha) \nabla \cdot D(\mathbf{u})+\nabla p=\mathbf{f}, \quad \text { in } \Omega \times(0, T), \\
& \lambda \sigma_{t}+\sigma+\lambda(\mathbf{u} \cdot \nabla) \sigma+\lambda g_{a}(\sigma, \nabla \mathbf{u})-2 \alpha D(\mathbf{u})=0, \quad \text { in } \Omega \times(0, T), \\
& \operatorname{div} \mathbf{u}=0, \quad \text { in } \Omega \times(0, T), \\
& \mathbf{u}=\mathbf{0}, \quad \text { on } \Gamma \times(0, T), \\
& \mathbf{u}(0, x)=\mathbf{u}_{0}(x), \quad \sigma(0, x)=\sigma_{0}(x), \quad \text { on } \Omega \times\{0\},
\end{aligned}
$$

where $\lambda$ is the Weissenberg number, Re is the Reynolds number, $0<\alpha<1$ may be considered as the fraction of viscoelastic viscosity ( $\alpha=1$ for Maxwell's model and this case is excluded here, see reference [23] for a description of various models), and $\mathbf{f}$ the body forces. The unknowns are the fluid velocity vector $\mathbf{u}=\left(u_{1}, u_{2}\right)$, the pressure $p$, and the stress $\sigma$, which is the viscoelastic part of the total stress tensor $\sigma_{\text {tot }}=\sigma+2(1-\alpha) D(\mathbf{u})-p \mathbf{I}$. In (2.1), $D(\mathbf{u})=(1 / 2)\left(\nabla \mathbf{u}+\nabla \mathbf{u}^{T}\right)$ is the rate of the strain tensor, for all $a \in[-1,1], g_{a}(\sigma, \nabla \mathbf{u})$ is defined by

$$
g_{a}(\sigma, \nabla \mathbf{u})=\frac{1-a}{2}\left(\sigma \nabla \mathbf{u}+(\nabla \mathbf{u})^{T} \sigma\right)-\frac{1+a}{2}\left((\nabla \mathbf{u}) \sigma+\sigma(\nabla \mathbf{u})^{T}\right)
$$

Note that an Oldroyd B constitutive model is obtained when $a=1$ in $g_{a}(\sigma, \nabla \mathbf{u})$.

The $L^{2}(\Omega)$ inner product is denoted by $(\cdot, \cdot)$, and the $L^{p}(\Omega)$ norm by $\|\cdot\|_{L^{p}}$, with the special cases of $L^{2}(\Omega)$ and $L^{\infty}(\Omega)$ norms being written as $\|\cdot\|$ and $\|\cdot\|_{\infty}$. For $k \in N$, we denote the norm associated with the Sobolev space $W^{m, p}(\Omega)$ by $\|\cdot\|_{W^{m, p}}$, with the special case $W^{m, 2}(\Omega)$ being written as $H^{m}(\Omega)$ with the norm $\|\cdot\|_{m}$ and seminorm $|\cdot|_{m}$. In order to introduce a variational formulation, we set the spaces $X, Q, S, V$ as follows:

$$
\begin{aligned}
X:= & H_{0}^{1}(\Omega)^{2}:=\left\{v \in H^{1}(\Omega)^{2}: \mathbf{v}=\mathbf{0} \text { on } \Gamma\right\}, \\
Q:= & L_{0}^{2}(\Omega)=\left\{q \in L^{2}(\Omega) ; \int_{\Omega} q d x=0\right\}, \\
S:= & \left\{\tau=\left(\tau_{i j}\right) ; \tau_{i j}=\tau_{j i} ; \tau_{i j} \in L^{2}(\Omega) ; i, j=1,2\right\} \\
& \cap\left\{\tau=\left(\tau_{i j}\right) ; \mathbf{v} \cdot \nabla \tau \in L^{2}(\Omega), \forall \mathbf{v} \in X\right\}, \\
V:= & \left\{\mathbf{v} \in X ; \int_{\Omega} q(\nabla \cdot \mathbf{v}) d x=0, \forall q \in Q\right\} .
\end{aligned}
$$


The corresponding weak formulation of problem (2.1) is then obtained: find $(\sigma, \mathbf{u}, p) \in$ $(S, X, Q)$ such that for all $(\tau, \mathbf{v}, q) \in(S, X, Q)$

$$
\begin{aligned}
& \left(\operatorname{Re} \mathbf{u}_{t}+\operatorname{Re} \mathbf{u} \cdot \nabla \mathbf{u}, \mathbf{v}\right)+(\sigma, D(\mathbf{v}))+2(1-\alpha)(D(\mathbf{u}), D(\mathbf{v}))+(p, \nabla \cdot \mathbf{v})=(\mathbf{f}, \mathbf{v}) \\
& \lambda\left(\sigma_{t}, \tau\right)+(\sigma, \tau)+\lambda((\mathbf{u} \cdot \nabla) \sigma, \tau)+\lambda\left(g_{a}(\sigma, \nabla \mathbf{u}), \tau\right)-2 \alpha(D(\mathbf{u}), \tau)=\mathbf{0} \\
& (q, \nabla \cdot \mathbf{u})=0
\end{aligned}
$$

Using the weak divergence free space $V$, the weak formulation (2.4) can be written as follows: find $(\sigma, \mathbf{u}) \in(S, V)$ such that for all $(\tau, \mathbf{v}) \in(S, V)$

$$
\begin{aligned}
\left(\operatorname{Re} \mathbf{u}_{t}+\operatorname{Re} \mathbf{u} \cdot \nabla \mathbf{u}, \mathbf{v}\right)+(\sigma, D(\mathbf{v}))+2(1-\alpha)(D(\mathbf{u}), D(\mathbf{v})) & =(\mathbf{f}, \mathbf{v}), \\
\lambda\left(\sigma_{t}, \tau\right)+(\sigma, \tau)+\lambda((\mathbf{u} \cdot \nabla) \sigma, \tau)+\lambda\left(g_{a}(\sigma, \nabla \mathbf{u}), \tau\right)-2 \alpha(D(\mathbf{u}), \tau) & =\mathbf{0} .
\end{aligned}
$$

Existence results for problem (2.1) are proved in [24] for the "slow flow" model of (2.1) (i.e., the $\mathbf{u} \cdot \nabla \mathbf{u}$ term in momentum equation is ignored). They are of two kinds: local existence in time of strong solutions in space variable in a $C^{3}$ domain, and global existence (in time) of a unique solution for $\mathbf{u}$ and $\sigma$ under a small data assumption on $\mathbf{f}, \mathbf{f}_{t}, \mathbf{u}_{0}, \sigma_{0}$. For a more complete discussion of existence and uniqueness issues, see [25].

\section{Finite Element Approximation}

In this section, we present a fully discrete approximation to (2.5). We begin by describing the finite element approximation framework.

Suppose $T^{h}$ is a uniformly regular triangulation of $\Omega$ such that $\Omega=\{\cup K: K \in$ $\left.T^{h}\right\}$ and assume that there exist positive constants $C_{1}, C_{2}$ such that $C_{1} h \leqslant h_{K} \leqslant C_{2} \rho_{K}$, where $h_{K}$ is the diameter of $K, \rho_{K}$ is the diameter of the greatest ball included in $K$, and $h=\max _{K \in T^{h}} h_{K}$. Throughout the paper, the constants $C, C_{1}, C_{2}, \ldots$ denote different constants which are independent of mesh size $h$ and time step $k$. We use the classical Taylor-Hood FE for the approximation in space of $(\mathbf{u}, p): P_{2}$-continuous in velocity, $P_{1}$-continuous in pressure; we consider $P_{1}$-continuous approximation of the stress $\sigma$. The corresponding FE spaces are

$$
\begin{aligned}
X^{h} & =\left\{\mathbf{v} \in X \cap C^{0}(\Omega)^{2} ; \mathbf{v}_{\mid K} \in P_{2}(K)^{2}, \forall K \in T^{h}\right\}, \\
S^{h} & =\left\{\tau \in S \cap C^{0}(\Omega)^{2 \times 2} ; \tau_{\mid K} \in P_{1}(K)^{2 \times 2} ; \forall K \in T^{h}\right\}, \\
Q^{h} & =\left\{q \in Q \cap C^{0}(\Omega) ; q_{\mid K} \in P_{1}(K) ; \forall K \in T^{h}\right\}, \\
V^{h} & =\left\{\mathbf{v} \in X^{h} ;(q, \nabla \cdot v)=0, \forall q \in Q^{h}\right\},
\end{aligned}
$$

where $P_{w}(K)$ denotes the space of polynomials of degree $\leqslant w$ on $K \in T^{h}$. It is well known $[26,27]$ that the Taylor-Hood pair $\left(X^{h}, Q^{h}\right)$ satisfies the discrete inf-sup (or LBB) condition.

To obtain the fully discrete approximation, the time derivatives are replaced by backward Euler differences, and the nonlinear terms are lagged. In order to stabilize 
the hyperbolic constitutive equation, an SUPG formulation is used to avoid spurious oscillations in the numerical approximation. Let $N$ be an integer, we divide the interval $[0, T]$ into $N$ intervals of equal length $k$ and denote $t_{n}=n k$. For notational convenience, we denote $v^{n}:=v\left(\cdot, t_{n}\right)$ and

$$
d_{t} g^{s}:=\frac{g\left(t_{s}\right)-g\left(t_{s-1}\right)}{k}, \quad b(\mathbf{u}, \sigma, \tau)=(\mathbf{u} \cdot \nabla \sigma, \tau), \quad c(\mathbf{u}, \mathbf{v}, \mathbf{w})=(\mathbf{u} \cdot \nabla \mathbf{v}, \mathbf{w})
$$

The following norms are used in the analysis:

$$
\left.\|g\|\right|_{\infty, m}:=\max _{1 \leqslant n \leqslant N}\left\|g^{n}\right\|_{m^{\prime}} \quad|\|g\||_{0, m}:=\left[\sum_{n=1}^{N} k\left\|g^{n}\right\|_{m}^{2}\right]^{1 / 2}
$$

and when $\psi(\mathbf{x}, t)$ is defined on the entire time interval $(0, T)$, we denote

$$
\|\psi\|_{\infty, m}:=\sup _{0 \leqslant t \leqslant T}\|\varphi(\cdot, t)\|_{m^{\prime}} \quad\|\psi\|_{0, m}^{2}:=\int_{0}^{T}\|\psi(\cdot, t)\|_{m}^{2} d t, \quad\|\psi\|(t)=\|\psi(\cdot, t)\| .
$$

Then, based on SUPG formulation, the fully discrete approximating systems of (2.5) is the following;

given $\mathbf{u}^{h, 0}=\mathbf{u}_{0}, \ldots, \mathbf{u}^{h, n} ; \sigma^{h, 0}=\sigma_{0}, \ldots, \sigma^{h, n}, n=0,1,2, \ldots, N-1$, find $\left(u^{h, n+1}, \sigma^{h, n+1}\right) \in$ $\left(V^{h}, S^{h}\right)$ such that for all $(\mathbf{v}, \tau) \in\left(V^{h}, S^{h}\right)$

$$
\begin{aligned}
& \operatorname{Re}\left(d_{t} \mathbf{u}^{h, n+1}, \mathbf{v}\right)+\operatorname{Re} c\left(\mathbf{u}^{h, n}, \mathbf{u}^{h, n+1}, \mathbf{v}\right)+2(1-\alpha)\left(D\left(\mathbf{u}^{h, n+1}\right), D(\mathbf{v})\right)+\left(\sigma^{h, n+1}, D(\mathbf{v})\right) \\
& \quad=\left(\mathbf{f}^{n+1}, \mathbf{v}\right) \\
& \lambda\left(d_{t} \sigma^{h, n+1}, \tau\right)+\left(\sigma^{h, n+1}, \bar{\tau}\right)+\lambda b\left(\mathbf{u}^{h, n}, \sigma^{h, n+1}, \bar{\tau}\right)-2 \alpha\left(D\left(\mathbf{u}^{h, n+1}\right), \bar{\tau}\right)=-\lambda\left(g_{a}\left(\sigma^{h, n}, \nabla \mathbf{u}^{h, n}\right), \bar{\tau}\right),
\end{aligned}
$$

where $\bar{\tau}=\tau+v \tau^{n, u}, \tau^{n, u}=u^{h, n} \cdot \nabla \tau, v$ is a small positive constant.

The goal of the parameter $v$ is used to suppress the production of spurious oscillations in the approximation. The discretization of the constitutive equation is the usual Galerkin finite element method if $v=0$.

The existence of a unique solution and a priori error estimate to (3.5) can be found in $[2,3,5]$.

\section{Defect Correction Method}

In this section, our defect correction method used in computing the solution to (3.5) is described as follows. 
Algorithm 1 (Defect-correction method).

Step 1. Solve the defected problem: find $\left(\mathbf{u}_{1}^{h, n+1}, \sigma_{1}^{h, n+1}\right) \in\left(V^{h}, S^{h}\right)$ such that for all $(\mathbf{v}, \tau) \in$ $\left(V^{h}, S^{h}\right)$

$$
\begin{aligned}
& \operatorname{Re}\left(d_{t} \mathbf{u}_{1}^{h, n+1}, \mathbf{v}\right)+\operatorname{Re} c\left(\mathbf{u}_{1}^{h, n}, \mathbf{u}_{1}^{h, n+1}, \mathbf{v}\right)+2(1-\alpha)\left(D\left(\mathbf{u}_{1}^{h, n+1}\right), D(\mathbf{v})\right)+\left(\sigma_{1}^{h, n+1}, D(\mathbf{v})\right) \\
& \quad=\left(\mathbf{f}^{n+1}, \mathbf{v}\right), \\
& \lambda\left(d_{t} \sigma_{1}^{h, n+1}, \tau\right)+\left(\sigma_{1}^{h, n+1}, \bar{\tau}\right)+\lambda_{1} b\left(\mathbf{u}_{1}^{h, n}, \sigma_{1}^{h, n+1}, \bar{\tau}\right)-2 \alpha\left(D\left(\mathbf{u}_{1}^{h, n+1}\right), \bar{\tau}\right) \\
& =-\lambda\left(g_{a}\left(\sigma_{1}^{h, n}, \nabla \mathbf{u}_{1}^{h, n}\right), \bar{\tau}\right),
\end{aligned}
$$

where $E$ is chosen such that $\lambda_{1}=\lambda-E h>0$.

Step 2. For $i=1,2, \ldots$, solve the correction problem: find $\left(\mathbf{u}_{i+1}^{h, n+1}, \sigma_{i+1}^{h, n+1}\right) \in\left(V^{h}, S^{h}\right)$ such that for all $(\mathbf{v}, \tau) \in\left(V^{h}, S^{h}\right)$

$$
\begin{aligned}
& \operatorname{Re}\left(d_{t} \mathbf{u}_{i+1}^{h, n+1}, \mathbf{v}\right)+\operatorname{Re} c\left(\mathbf{u}_{i+1}^{h, n}, \mathbf{u}_{i+1}^{h, n+1}, \mathbf{v}\right)+2(1-\alpha)\left(D\left(\mathbf{u}_{i+1}^{h, n+1}\right), D(\mathbf{v})\right)+\left(\sigma_{i+1}^{h, n+1}, D(\mathbf{v})\right) \\
& =\left(\mathbf{f}^{n+1}, \mathbf{v}\right), \\
& \lambda\left(d_{t} \sigma_{i+1}^{h, n+1}, \tau\right)+\left(\sigma_{i+1}^{h, n+1}, \bar{\tau}\right)+\lambda_{1} b\left(\mathbf{u}_{i+1}^{h, n}, \sigma_{i+1}^{h, n+1}, \bar{\tau}\right)-2 \alpha\left(D\left(\mathbf{u}_{i+1}^{h, n+1}\right), \bar{\tau}\right) \\
& =-\lambda\left(g_{a}\left(\sigma_{i+1}^{h, n}, \nabla \mathbf{u}_{i+1}^{h, n}\right), \bar{\tau}\right)-\left(\lambda-\lambda_{1}\right) b\left(\mathbf{u}_{i+1}^{h, n}, \sigma_{i}^{h, n+1}, \bar{\tau}\right) .
\end{aligned}
$$

The initial value approximations are taken to be $\mathbf{u}_{1}^{h, 0}=\mathbf{u}_{i+1}^{h, 0}=\mathbf{u}_{0}$, and $\sigma_{1}^{h, 0}=\sigma_{i+1}^{h, 0}=\sigma_{0}$. In order to ensure computability of the algorithm, we begin by showing that (4.1)-(4.2) and (4.3)-(4.4) are uniquely solvable for $\left(\mathbf{u}_{j}^{h, n+1}, \sigma_{j}^{h, n+1}\right), j=1,2$ at each time step $n+1$. We use the following induction hypothesis, which simply states that the computed iterates $\left(\mathbf{u}_{j}^{h, n+1}, \sigma_{j}^{h, n+1}\right), j=1,2$ are bounded independent of $h$ and $n$ :

$$
\text { (IH1) : }\left\|u_{j}^{h, s}\right\|_{\infty}, \quad\left\|\sigma_{j}^{h, s}\right\|_{\infty} \leqslant K, \quad j=1,2,0 \leqslant s \leqslant n
$$

In next section, we will prove the induction hypothesis (IH1) is right.

Lemma 4.1. Suppose (IH1) is true. For sufficiently small step size $k$, then Step 1 of Algorithm 1 admits a unique solution $\left(u_{1}^{h, n+1}, \sigma_{1}^{h, n+1}\right) \in\left(V^{h}, S^{h}\right)$.

Proof. Letting $\mathbf{v}=\mathbf{u}_{1}^{h, n+1}, \tau=\sigma_{1}^{h, n+1}$, multiplying (4.1) by $2 \alpha$, and adding to (4.2), we get

$$
\begin{aligned}
A\left(\mathbf{u}_{1}^{h, n+1}, \sigma_{1}^{h, n+1} ; \mathbf{u}_{1}^{h, n+1}, \sigma_{1}^{h, n+1}\right)= & 2 \alpha\left(f^{n+1}, \mathbf{u}_{1}^{h, n+1}\right)+\frac{2 \alpha \operatorname{Re}}{k}\left(\mathbf{u}_{1}^{h, n}, \mathbf{u}_{1}^{h, n+1}\right) \\
& -\lambda\left(g_{a}\left(\sigma_{1}^{h, n}, \nabla \mathbf{u}_{1}^{h, n}\right), \bar{\sigma}_{1}^{h, n+1}\right)+\frac{\lambda}{k}\left(\sigma_{1}^{h, n}, \sigma_{1}^{h, n+1}\right),
\end{aligned}
$$


where the bilinear form $A(\mathbf{u}, \sigma ; \mathbf{v}, \tau)$ is defined by

$$
\begin{aligned}
A(\mathbf{u}, \sigma ; \mathbf{v}, \tau)= & \frac{2 \alpha \operatorname{Re}}{k}(\mathbf{u}, \mathbf{v})+2 \alpha \operatorname{Re} c\left(\mathbf{u}^{n}, \mathbf{u}, \mathbf{v}\right)+4 \alpha(1-\alpha)(D(\mathbf{u}), D(\mathbf{v})) \\
& +2 \alpha(\sigma, D(\mathbf{v}))+(\sigma, \bar{\tau})+\frac{\lambda}{k}(\sigma, \tau)+\lambda_{1} b\left(\mathbf{u}^{n}, \sigma, \tau\right) \\
& +\lambda_{1} b\left(\mathbf{u}^{n}, \sigma, v u^{n} \cdot \nabla \tau\right)-2 \alpha(D(\mathbf{u}), \sigma)-2 \alpha\left(D(\mathbf{u}), v u^{n} \cdot \nabla \tau\right)
\end{aligned}
$$

As (4.1)-(4.2) represent a finite system of liner equations, the positivity of $A\left(\mathbf{u}_{1}^{h, n+1}, \sigma_{1}^{h, n+1}\right.$; $\left.\mathbf{u}_{1}^{h, n+1}, \sigma_{1}^{h, n+1}\right)$ is a sufficient condition for the existence and uniqueness of $\left(\mathbf{u}_{1}^{h, n+1}, \sigma_{1}^{h, n+1}\right)$.

We now estimate each term of $A\left(\mathbf{u}_{1}^{h, n+1}, \sigma_{1}^{h, n+1} ; \mathbf{u}_{1}^{h, n+1}, \sigma_{1}^{h, n+1}\right)$. We have

$$
\begin{aligned}
\left|2 \alpha \operatorname{Re} c\left(\mathbf{u}_{1}^{h, n}, \mathbf{u}_{1}^{h, n+1}, \mathbf{u}_{1}^{h, n+1}\right)\right| & \leq \epsilon_{1}\left\|D\left(\mathbf{u}_{1}^{h, n+1}\right)\right\|^{2}+\frac{\alpha^{2} d^{\prime} K^{2} C_{K}^{2} \operatorname{Re}^{2}}{\epsilon_{1}}\left\|\mathbf{u}_{1}^{h, n+1}\right\|^{2}, \\
\left|\left(\sigma_{1}^{h, n+1}, v \sigma_{1}^{n, u}\right)\right| & \leq\left\|\sigma_{1}^{h, n+1}\right\|_{0}^{2}+\frac{v^{2}}{4}\left\|\sigma_{1}^{n, u}\right\|^{2}, \\
\left|\lambda_{1} b\left(\mathbf{u}_{1}^{h, n}, \sigma_{1}^{h, n+1}, \sigma_{1}^{h, n+1}\right)\right| & \leq \epsilon_{2}\left\|\sigma_{1}^{n, u}\right\|^{2}+\frac{\lambda_{1}^{2}}{4 \epsilon_{2}}\left\|\sigma_{1}^{h, n+1}\right\|^{2}, \\
\lambda_{1} b\left(\mathbf{u}_{1}^{h, n}, \sigma_{1}^{h, n+1}, v \sigma_{1}^{n, u}\right) & =\lambda_{1} v\left\|\sigma_{1}^{n, u}\right\|^{2}, \\
\left|2 \alpha\left(D\left(\mathbf{u}_{1}^{h, n+1}\right), v \sigma_{1}^{n, u}\right)\right| & \leq \epsilon_{3}\left\|D\left(\mathbf{u}_{1}^{h, n+1}\right)\right\|^{2}+\frac{\alpha^{2} v^{2}}{\epsilon_{3}}\left\|\sigma_{1}^{n, u}\right\|^{2},
\end{aligned}
$$

where we have used the induction hypothesis (IH1) and the Korn's lemma, $C_{K}$ is Korn constant.

Substituting all above equations into $A(\cdot, \cdot ; \cdot, \cdot)$, we have

$$
\begin{aligned}
& A\left(\mathbf{u}_{1}^{h, n+1}, \sigma_{1}^{h, n+1}, \mathbf{u}_{1}^{h, n+1}, \sigma_{1}^{h, n+1}\right) \\
& \quad \geq\left(\frac{2 \alpha \operatorname{Re}}{k}-\frac{\alpha^{2} d^{\prime} K^{2} C_{K}^{2} \operatorname{Re}^{2}}{\epsilon_{1}}\right)\left\|\mathbf{u}_{1}^{h, n+1}\right\|^{2}+\left(\frac{\lambda}{k}-\frac{\lambda_{1}^{2}}{4 \epsilon_{2}}\right)\left\|\sigma_{1}^{h, n+1}\right\|^{2} \\
& \quad+\left(4 \alpha(1-\alpha)-\epsilon_{1}-\epsilon_{3}\right)\left\|D\left(\mathbf{u}_{1}^{h, n+1}\right)\right\|^{2}+\left(\lambda_{1} v-\epsilon_{2}-\frac{v^{2}}{4}-\frac{\alpha^{2} v^{2}}{\epsilon_{3}}\right)\left\|\sigma_{1}^{n, u}\right\|^{2}
\end{aligned}
$$

Choosing $v \leq\left(2 \lambda_{1}(1-\alpha)\right) /(1+3 \alpha), k \leq \min \left\{2 \lambda v / \lambda_{1}, 2(1-\alpha) / d^{\prime} K^{2} C_{K}^{2} \operatorname{Re}\right\}$ and $\epsilon_{1}=\epsilon_{3}=\alpha(1-$ $\alpha), \epsilon_{2}=\lambda_{1} v / 2$, the bilinear form $A(\cdot, \cdot ; \cdot, \cdot)$ is positive definite. Hence, for $k$ chosen sufficiently small, Step 1 of Algorithm 1 admits a unique solution $\left(u_{1}^{h, n+1}, \sigma_{1}^{h, n+1}\right) \in\left(V^{h}, S^{h}\right)$.

Similarity as Lemma 4.1, we can derive that Step 2 of Algorithm 1 admits a unique solution $\left(u_{2}^{h, n+1}, \sigma_{2}^{h, n+1}\right) \in\left(V^{h}, S^{h}\right)$. 


\section{A Priori Error Estimates}

In this section, we explore the error estimates in approximating the true solution $(\mathbf{u}, p, \sigma)$ of (2.1) by the defect step approximation $\left(u_{1}, p_{1}, \sigma_{1}\right)$ and one correction step approximation $\left(u_{2}, p_{2}, \sigma_{2}\right)$. The main results of this section are presented in the following theorem.

Theorem 5.1. Suppose that (2.1) has a solution $(\mathbf{u}, \sigma, p) \in C^{2}\left(0, T ; H^{3}\right) \times C^{2}\left(0, T ; H^{2}\right) \times$ $C^{1}\left(0, T ; H^{2}\right)$. In addition, assume that $k, v \leqslant c h^{d^{\prime} / 2}$, and $\|\mathbf{u}\|_{\infty},\|\nabla \mathbf{u}\|_{\infty},\|\sigma\|_{\infty},\|\nabla \sigma\|_{\infty} \leqslant M$ for all $t \in[0, T],\left(\mathbf{u}_{1}^{h}, \sigma_{1}^{h}\right)$ and $\left(\mathbf{u}_{2}^{h}, \sigma_{2}^{h}\right)$ satisfy (4.1)-(4.2) and (4.3)-(4.4), respectively. Then, there exists a constant $C=C\left(\operatorname{Re}, \alpha, \Omega, T, \mathbf{u}, p, \sigma, \mathbf{f}, \lambda_{1}, \lambda\right)$, such that

$$
\begin{aligned}
& ||\left|\mathbf{u}-\mathbf{u}_{1}^{h}\left\|\left.\right|_{\infty, 0}+\left|\left\|\sigma-\sigma_{1}^{h}\right\|\right|_{\infty, 0}+\left|\left\|\mathbf{u}-\mathbf{u}_{1}^{h}\right\|\right|_{0,1}+\left|\left\|\sigma-\sigma_{1}^{h}\right\|\right|_{0,0} \leqslant B(k, h, v),\right.\right. \\
& \left|\left\|\mathbf{u}-\mathbf{u}_{2}^{h}\right\|\right|_{\infty, 0}+\left|\left\|\sigma-\sigma_{2}^{h}\right\|\right|_{\infty, 0}+\left|\left\|\mathbf{u}-\mathbf{u}_{2}^{h}\right\|\right|_{0,1}+\left|\left\|\sigma-\sigma_{2}^{h}\right\|\right|_{0,0} \leqslant B(k, h, v),
\end{aligned}
$$

where

$$
\begin{aligned}
B(k, h, v)= & C\left(h^{2}|\|\mathbf{u}\||_{0,3}+h^{3}\left|\left\|\mathbf{u}_{t}\right\|\right|_{0,3}+h|\|\sigma\||_{0,2}+h^{2}\left|\left\|\sigma_{t}||_{0,2}+h^{2}|\|p\||_{0,2}\right)\right.\right. \\
& +C\left(h^{3}|\|\mathbf{u}\||_{\infty, 3}+h^{2}|\|\sigma\||_{\infty, 2}\right)+C k\left(\left\|\mathbf{u}_{t}\right\|_{0,1}+\left\|\mathbf{u}_{t t}\right\|_{0,0}\right. \\
& \left.+\left\|\sigma_{t}\right\|_{0,1}+\left\|\sigma_{t t}\right\|_{0,0}\right)+C v\left(\left|\left\|\sigma_{t}\right\|\right|_{0,1}+\left|\left\|\sigma_{t}\right\|\right|_{\infty, 0}\right)+C \sqrt{T}\left(\lambda-\lambda_{1}\right) .
\end{aligned}
$$

Remark 5.2. As mentioned in Algorithm $1, \lambda-\lambda_{1}=E h$, thus, for $i=1,2$, the essence of the estimates (5.1) and (5.2) is

$$
\left|\left\|\mathbf{u}-\mathbf{u}_{i}^{h}\right\|\right|_{\infty, 0}+\left|\left\|\sigma-\sigma_{i}^{h}\right\|\right|_{\infty, 0}+\left|\left\|\mathbf{u}-\mathbf{u}_{i}^{h}\right\|\right|_{0,1}+\left|\left\|\sigma-\sigma_{i}^{h}\right\|\right|_{0,0} \leqslant C(h+k+v) .
$$

Before deriving the estimates (5.2), (5.9), we first introduce some notation and some approximating properties of finite element spaces. Let $\mathbf{u}^{n}=\mathbf{u}\left(t_{n}\right), \sigma^{n}=\sigma\left(t_{n}\right)$ represent the solution of $(2.5)$ at time $t_{n}=n k$. Assume $(\mathbf{u}, \sigma, p) \in C^{2}\left(0, T ; H^{3}\right) \times C^{2}\left(0, T ; H^{2}\right) \times$ $C^{1}\left(0, T ; H^{2}\right)$. Let $\left(\widetilde{\mathbf{u}}^{n}, \tilde{p}^{n}\right)$ denote the Stokes projection of $\left(\mathbf{u}^{n}, p^{n}\right)$ into $\left(V^{h}, Q^{h}\right)$, and $\widetilde{\sigma}^{n}$ a Clement interpolant of $\sigma^{n}[26,28]$. The following approximating properties are right.

$$
\begin{gathered}
\left\|\mathbf{u}^{n}-\widetilde{\mathbf{u}}^{n}\right\|+h\left\|\nabla\left(\mathbf{u}^{n}-\widetilde{\mathbf{u}}^{n}\right)\right\| \leqslant C h^{3}\left\|\mathbf{u}^{n}\right\|_{3}, \\
\left\|p^{n}-\widetilde{p}^{n}\right\| \leqslant C h^{2}\left\|p^{n}\right\|_{2^{\prime}} \\
\left\|\sigma^{n}-\widetilde{\sigma}^{n}\right\|+h\left\|\nabla\left(\sigma^{n}-\tilde{\sigma}^{n}\right)\right\| \leqslant C h^{2}\left\|\sigma^{n}\right\|_{2} .
\end{gathered}
$$


Let $\left(\mathbf{u}_{1}^{h, n}, \sigma_{1}^{h, n}\right)$ and $\left(\mathbf{u}_{2}^{h, n}, \sigma_{2}^{h, n}\right)$ denote the solutions of (4.1)-(4.2) and (4.3)-(4.4), respectively. We denote $\Phi_{j}^{n}, Y_{j}^{n}, e_{j}^{n}, \Psi_{j}^{n}, U_{j}^{n}, \epsilon_{j}^{n}, j=1,2$ as follows:

$$
\begin{array}{lll}
\Phi_{j}^{n}=\mathbf{u}^{n}-\widetilde{\mathbf{u}}^{n}, & Y_{j}^{n}=\widetilde{\mathbf{u}}^{n}-\mathbf{u}_{j}^{h, n}, \quad e_{j}^{n}=\Phi_{j}^{n}+Y_{j}^{n}=\mathbf{u}^{n}-\mathbf{u}_{j}^{h, n}, \\
\Psi_{j}^{n}=\sigma^{n}-\widetilde{\sigma}^{n}, & U_{j}^{n}=\widetilde{\sigma}^{n}-\sigma_{j}^{h, n}, \quad \epsilon_{j}^{n}=\Psi_{j}^{n}+U_{j}^{n}=\sigma^{n}-\sigma_{j}^{h, n} .
\end{array}
$$

In order to establish Theorem 5.1, we need another induction hypothesis, that is,

$$
\text { (IH2) : } \sum_{n=0}^{s-1} k\left\|\nabla Y_{j}^{n}\right\|_{\infty} \leqslant 1, \quad j=1,2
$$

The induction hypothesis (IH2) will be proved later.

Proof. We first give the profile of the proof. The proof of Theorem 5.1 is established in two steps.

Step 1. Prove the error estimate (5.1) is right. We divide Step 1 into two substep.

Substep 1.1. Under the induction hypothesis (IH1) and (IH2) for $j=1$, we prove the error estimate (5.1) is true.

Substep 1.2. We prove that the two induction hypotheses (IH1) and (IH2) for $j=1$ are right.

Step 2. Show that the error estimate (5.2) is true.

Now, we start to prove Theorem 5.1.

Step 1. Prove the error estimate (5.1) is right.

Substep 1.1. Under the induction hypotheses (IH1) and (IH2), we prove the error estimate (5.1) is true.

As $(\sigma, \mathbf{u}, p)$ being the exact solution of (2.1), it satisfies the following consistency equation: for all $(\tau, \mathbf{v}) \in S^{h} \times V^{h}$, in particular, at time $t=t_{n+1}$ (noting that we denote $\left.v^{n+1}:=v\left(\cdot, t_{n+1}\right)\right)$,

$$
\begin{aligned}
2 \alpha \operatorname{Re}\left(\mathbf{u}_{t}^{n+1}, v\right)+\left(\lambda \sigma_{t}^{n+1}, \bar{\tau}\right)+2 \alpha c\left(\operatorname{Re} \mathbf{u}^{n+1}, \mathbf{u}^{n+1}, \mathbf{v}\right) \\
+2 \alpha\left(\sigma^{n+1}, D(\mathbf{v})\right)+4 \alpha(1-\alpha)\left(D\left(\mathbf{u}^{n+1}\right), D(\mathbf{v})\right) \\
+\lambda b\left(\mathbf{u}^{n+1}, \sigma^{n+1}, \bar{\tau}\right)+\left(\sigma^{n+1}, \bar{\tau}\right)-2 \alpha\left(D\left(\mathbf{u}^{n+1}\right), \bar{\tau}\right) \\
=2 \alpha\left(f^{n+1}, \mathbf{v}\right)+2 \alpha\left(p^{n+1}, \nabla \cdot \mathbf{v}\right)-\lambda\left(g_{a}\left(\sigma^{n+1}, \nabla \mathbf{u}^{n+1}\right), \bar{\tau}\right) .
\end{aligned}
$$


Equation (5.8) can also be written as

$$
\begin{aligned}
2 \alpha \operatorname{Re}\left(d_{t} \mathbf{u}^{n+1}, \mathbf{v}\right)+\left(\lambda d_{t} \sigma^{n+1}, \tau\right)+2 \alpha c\left(\operatorname{Re} \mathbf{u}_{1}^{h, n}, \mathbf{u}^{n+1}, \mathbf{v}\right) \\
\quad+4 \alpha(1-\alpha)\left(D\left(\mathbf{u}^{n+1}\right), D(\mathbf{v})\right)+2 \alpha\left(\sigma^{n+1}, D(\mathbf{v})\right)+\lambda_{1} b\left(\mathbf{u}_{1}^{h, n}, \sigma^{n+1}, \bar{\tau}\right) \\
\quad+\left(\sigma^{n+1}, \bar{\tau}\right)-2 \alpha\left(D\left(u^{n+1}\right), \bar{\tau}\right) \\
=2 \alpha\left(\mathbf{f}^{n+1}, \mathbf{v}\right)+2 \alpha\left(p^{n+1}, \nabla \cdot \mathbf{v}\right)-\lambda\left(g_{a}\left(\sigma_{1}^{h, n}, \nabla \mathbf{u}_{1}^{h, n}\right), \bar{\tau}\right) \\
+2 \alpha \operatorname{Re}\left(d_{t} \mathbf{u}^{n+1}, \mathbf{v}\right)-2 \alpha \operatorname{Re}\left(\mathbf{u}_{t}^{n+1}, \mathbf{v}\right)+2 \alpha c\left(\operatorname{Re} \mathbf{u}_{1}^{h, n}, \mathbf{u}^{n+1}, \mathbf{v}\right) \\
\quad-2 \alpha c\left(\operatorname{Re} \mathbf{u}^{n+1}, \mathbf{u}^{n+1}, \mathbf{v}\right)+\lambda\left(g_{a}\left(\sigma_{1}^{h, n}, \nabla \mathbf{u}_{1}^{h, n}\right), \bar{\tau}\right)-\lambda\left(g_{a}\left(\sigma^{n+1}, \nabla \mathbf{u}^{n+1}\right), \bar{\tau}\right) \\
+\left(\lambda d_{t} \sigma^{n+1}, \tau\right)-\left(\lambda \sigma_{t}^{n+1}, \bar{\tau}\right)+\lambda_{1} b\left(\mathbf{u}_{1}^{h, n}, \sigma^{n+1}, \bar{\tau}\right)-\lambda b\left(\mathbf{u}^{n+1}, \sigma^{n+1}, \bar{\tau}\right) .
\end{aligned}
$$

Multiplying (4.1) by $2 \alpha$ and adding to (4.2), for all $(\tau, \mathbf{v}) \in S^{h} \times V^{h}$, we get

$$
\begin{aligned}
2 \alpha \operatorname{Re}( & \left.d_{t} \mathbf{u}_{1}^{h, n+1}, \mathbf{v}\right)+\left(\lambda d_{t} \sigma_{1}^{h, n+1}, \tau\right)+2 \alpha c\left(\operatorname{Re} \mathbf{u}_{1}^{h, n}, \mathbf{u}_{1}^{h, n+1}, \mathbf{v}\right) \\
& +2 \alpha\left(\sigma_{1}^{h, n+1}, D(\mathbf{v})\right)+4 \alpha(1-\alpha)\left(D\left(\mathbf{u}_{1}^{h, n+1}\right), D(\mathbf{v})\right) \\
& +\lambda_{1} b\left(\mathbf{u}_{1}^{h, n}, \sigma_{1}^{h, n+1}, \bar{\tau}\right)+\left(\sigma_{1}^{h, n+1}, \bar{\tau}\right)-2 \alpha\left(D\left(\mathbf{u}_{1}^{h, n+1}\right), \bar{\tau}\right) \\
= & 2 \alpha\left(f^{n+1}, \mathbf{v}\right)-\lambda\left(g_{a}\left(\sigma_{1}^{h, n}, \nabla \mathbf{u}_{1}^{h, n}\right), \bar{\tau}\right) .
\end{aligned}
$$

Subtracting (5.9) from (5.10), for all $(\tau, \mathbf{v}) \in S^{h} \times V^{h}$, we obtain the following equation for $e_{1}^{n+1}$ and $\epsilon_{1}^{n+1}$ :

$$
\begin{aligned}
2 \alpha \operatorname{Re}( & \left.d_{t} e_{1}^{n+1}, \mathbf{v}\right)+\left(\lambda d_{t} \epsilon_{1}^{n+1}, \tau\right)+2 \alpha c\left(\operatorname{Re} \mathbf{u}_{1}^{h, n}, e_{1}^{n+1}, \boldsymbol{v}\right) \\
& +4 \alpha(1-\alpha)\left(D\left(e_{1}^{n+1}\right), D(\mathbf{v})\right)+2 \alpha\left(\epsilon_{1}^{n+1}, D(\mathbf{v})\right)+\lambda_{1} b\left(\mathbf{u}_{1}^{h, n}, \epsilon_{1}^{n+1}, \bar{\tau}\right) \\
& +\left(\epsilon_{1}^{n+1}, \bar{\tau}\right)-2 \alpha\left(D\left(e_{1}^{n+1}\right), \bar{\tau}\right) \\
= & 2 \alpha \operatorname{Re}\left(d_{t} \mathbf{u}^{n+1}-\mathbf{u}_{t}^{n+1}, \mathbf{v}\right)+2 \alpha \operatorname{Re} c\left(\mathbf{u}_{1}^{h, n}-\mathbf{u}^{n+1}, u^{n+1}, \mathbf{v}\right) \\
& -\left(\lambda \sigma_{t}^{n+1}, \bar{\tau}\right)+\left(\lambda d_{t} \sigma^{n+1}, \tau\right)-\lambda b\left(\mathbf{u}^{n+1}, \sigma^{n+1}, \bar{\tau}\right)+\lambda_{1} b\left(\mathbf{u}_{1}^{h, n}, \sigma^{n+1}, \bar{\tau}\right) \\
& +\lambda\left(g_{a}\left(\sigma_{1}^{h, n}, \nabla \mathbf{u}_{1}^{h, n}\right), \bar{\tau}\right)-\lambda\left(g_{a}\left(\sigma^{n+1}, \nabla \mathbf{u}^{n+1}\right), \bar{\tau}\right)+2 \alpha\left(p^{n+1}, \nabla \cdot \mathbf{v}\right) .
\end{aligned}
$$


Discrete Dynamics in Nature and Society

Substituting $e_{1}^{n+1}=Y_{1}^{n+1}+\Phi_{1}^{n+1}, \epsilon_{1}^{n+1}=U_{1}^{n+1}+\Psi_{1}^{n+1}, \mathbf{v}=Y_{1}^{n+1}$ and $\tau=U_{1}^{n+1}$ into (5.11), we get

$$
\begin{aligned}
2 \alpha \operatorname{Re}\left(d_{t} Y_{1}^{n+1}, Y_{1}^{n+1}\right)+\lambda\left(d_{t} U_{1}^{n+1}, U_{1}^{n+1}\right)+2 \alpha\left(U_{1}^{n+1}, D\left(Y_{1}^{n+1}\right)\right) \\
\quad+2 \alpha c\left(\operatorname{Re} \mathbf{u}_{1}^{h, n}, Y_{1}^{n+1}, Y_{1}^{n+1}\right)+4 \alpha(1-\alpha)\left(D\left(Y_{1}^{n+1}\right), D\left(Y_{1}^{n+1}\right)\right) \\
\quad+\left(U_{1}^{n+1}, \bar{U}_{1}^{n+1}\right)+\lambda_{1} b\left(\mathbf{u}_{1}^{h, n}, U_{1}^{n+1}, \bar{U}_{1}^{n+1}\right)-2 \alpha\left(D\left(Y_{1}^{n+1}\right), \bar{U}_{1}^{n+1}\right) \\
=\mathbf{F}\left(Y_{1}^{n+1}, U_{1}^{n+1}\right),
\end{aligned}
$$

where

$$
\begin{aligned}
& \mathbf{F}\left(Y_{1}^{n+1}, U_{1}^{n+1}\right) \\
&= 2 \alpha\left(p^{n+1}, \nabla \cdot Y_{1}^{n+1}\right)+2 \alpha \operatorname{Re}\left(d_{t} \mathbf{u}^{n+1}-\mathbf{u}_{t}^{n+1}, Y_{1}^{n+1}\right)-\left(\lambda \sigma_{t}^{n+1}, \bar{U}_{1}^{n+1}\right) \\
&+2 \alpha \operatorname{Re} c\left(\mathbf{u}_{1}^{h, n}-\mathbf{u}^{n+1}, \mathbf{u}^{n+1}, Y_{1}^{n+1}\right)+\lambda\left(g_{a}\left(\sigma_{1}^{h, n}, \nabla \mathbf{u}_{1}^{h, n}\right), \bar{U}_{1}^{n+1}\right) \\
&+\left(\lambda d_{t} \sigma^{n+1}, U_{1}^{n+1}\right)-\lambda\left(g_{a}\left(\sigma^{n+1}, \nabla \mathbf{u}^{n+1}\right), \bar{U}_{1}^{n+1}\right)-2 \alpha c\left(\operatorname{Re} \mathbf{u}_{1}^{h, n}, \Phi_{1}^{n+1}, Y_{1}^{n+1}\right) \\
&-4 \alpha(1-\alpha)\left(D\left(\Phi_{1}^{n+1}\right), D\left(Y_{1}^{n+1}\right)\right)-2 \alpha\left(\Psi_{1}^{n+1}, D\left(Y_{1}^{n+1}\right)\right)-\left(\Psi_{1}^{n+1}, \bar{U}_{1}^{n+1}\right) \\
&-\lambda b\left(\mathbf{u}^{n+1}, \sigma^{n+1}, \bar{U}_{1}^{n+1}\right)+\lambda_{1} b\left(\mathbf{u}_{1}^{h, n}, \sigma^{n+1}, \bar{U}_{1}^{n+1}\right)-\lambda_{1} b\left(\mathbf{u}_{1}^{h, n}, \Psi_{1}^{n+1}, \bar{U}_{1}^{n+1}\right) \\
&+2 \alpha\left(D\left(\Phi_{1}^{n+1}\right), \bar{U}_{1}^{n+1}\right)-2 \alpha \operatorname{Re}\left(d_{t} \Phi_{1}^{n+1}, Y_{1}^{n+1}\right)-\lambda\left(d_{t} \Psi_{1}^{n+1}, U_{1}^{n+1}\right) .
\end{aligned}
$$

Using the identity $(a-b, a)=(1 / 2)\left(\|a\|^{2}-\|b\|^{2}+\|a-b\|^{2}\right)$, we obtain

$$
\begin{aligned}
\frac{\alpha \operatorname{Re}}{k}( & \left.\left\|Y_{1}^{n+1}\right\|^{2}-\left\|Y_{1}^{n}\right\|^{2}+\left\|Y_{1}^{n+1}-Y_{1}^{n}\right\|^{2}\right)+4 \alpha(1-\alpha)\left\|D\left(Y_{1}^{n+1}\right)\right\|^{2} \\
& +\frac{\lambda}{2 k}\left(\left\|U_{1}^{n+1}\right\|^{2}-\left\|U_{1}^{n}\right\|^{2}+\left\|U_{1}^{n+1}-U_{1}^{n}\right\|^{2}\right)+\left\|U_{1}^{n+1}\right\|^{2}+v \lambda_{1}\left\|U_{1}^{n, u}\right\|^{2} \\
\leqslant & 2 \alpha \operatorname{Re}\left|c\left(\mathbf{u}_{1}^{h, n}, Y_{1}^{n+1}, Y_{1}^{n+1}\right)\right|+v\left|\left(U_{1}^{n+1}, U_{1}^{n, u}\right)\right|+\lambda_{1}\left|b\left(\mathbf{u}_{1}^{h, n}, U_{1}^{n+1}, U_{1}^{n+1}\right)\right| \\
& +2 \alpha v\left|\left(D\left(Y_{1}^{n+1}\right), U_{1}^{n, u}\right)\right|+\mathbf{F}\left(Y_{1}^{n+1}, U_{1}^{n+1}\right) .
\end{aligned}
$$


First multiply (5.14) by $k$ and sum it form $n=0$ to $s-1$, we obtain

$$
\begin{aligned}
& \alpha \operatorname{Re} \sum_{n=0}^{s-1}\left[\left\|Y_{1}^{n+1}\right\|^{2}-\left\|Y_{1}^{n}\right\|^{2}+\left\|Y_{1}^{n+1}-Y_{1}^{n}\right\|^{2}\right]+4 \alpha(1-\alpha) k \sum_{n=0}^{s-1}\left\|D\left(Y_{1}^{n+1}\right)\right\|^{2} \\
& \quad+\frac{\lambda}{2} \sum_{n=0}^{s-1}\left[\left\|\mathrm{U}_{1}^{n+1}\right\|^{2}-\left\|U_{1}^{n}\right\|^{2}+\left\|U_{1}^{n+1}-U_{1}^{n}\right\|^{2}\right]+k \sum_{n=0}^{s-1}\left\|U_{1}^{n+1}\right\|^{2}+v \lambda_{1} k \sum_{n=0}^{s-1}\left\|U_{1}^{n, u}\right\|^{2} \\
& \leqslant k \sum_{n=0}^{s-1}\left[2 \alpha \operatorname{Re}\left|c\left(\mathbf{u}_{1}^{h, n}, Y_{1}^{n+1}, Y_{1}^{n+1}\right)\right|+v\left|\left(U_{1}^{n+1}, U_{1}^{n, u}\right)\right|\right. \\
& \left.\quad+\lambda_{1}\left|b\left(\mathbf{u}_{1}^{h, n}, U_{1}^{n+1}, U_{1}^{n+1}\right)\right|+2 \alpha v \mid\left(D\left(Y_{1}^{n+1}\right), U_{1}^{n, u}\right) \|\right]+k \sum_{n=0}^{s-1} \mathrm{~F}\left(Y_{1}^{n+1}, U_{1}^{n+1}\right) .
\end{aligned}
$$

Then, we get, remarking that $Y_{1}^{0}=0$ and $U_{1}^{0}=0$,

$$
\begin{aligned}
& \alpha \operatorname{Re}\left[\left\|Y_{1}^{s}\right\|^{2}+\sum_{n=0}^{s-1}\left\|Y_{1}^{n+1}-Y_{1}^{n}\right\|^{2}\right]+\frac{\lambda}{2}\left[\left\|U_{1}^{s}\right\|^{2}+\sum_{n=0}^{s-1}\left\|U_{1}^{n+1}-U_{1}^{n}\right\|^{2}\right] \\
& +k \sum_{n=0}^{s-1}\left\|U_{1}^{n+1}\right\|^{2}+4 \alpha(1-\alpha) k \sum_{n=0}^{s-1}\left\|D\left(Y_{1}^{n+1}\right)\right\|^{2}+v \lambda_{1} k \sum_{n=0}^{s-1}\left\|U_{1}^{n, u}\right\|^{2} \\
& \leqslant k \sum_{n=0}^{s-1}\left[2 \alpha \operatorname{Re}\left|c\left(\mathbf{u}_{1}^{h, n}, Y_{1}^{n+1}, Y_{1}^{n+1}\right)\right|+v\left|\left(U_{1}^{n+1}, U_{1}^{n, u}\right)\right|\right. \\
& \left.+\lambda_{1}\left|b\left(\mathbf{u}_{1}^{h, n}, U_{1}^{n+1}, U_{1}^{n+1}\right)\right|+2 \alpha v \mid\left(D\left(Y_{1}^{n+1}\right), U_{1}^{n, u}\right) \|\right]+k \sum_{n=0}^{s-1} \mathbf{F}\left(Y_{1}^{n+1}, U_{1}^{n+1}\right) .
\end{aligned}
$$

For controlling each term on the right-hand side (RHS) of (5.16), the assumption (IH1) and (IH2) are needed here. We will prove the two induction hypotheses in the next subsection. Let us estimate each term of the RHS of (5.16). For details, please see $[2,3,6]$. We start from the first four terms of the RHS of (5.16);

$$
\begin{gathered}
2 \alpha \operatorname{Re}\left|c\left(\mathbf{u}_{1}^{h, n}, Y_{1}^{n+1}, Y_{1}^{n+1}\right)\right| \leqslant 4 \alpha^{2} \operatorname{Re}^{2} C_{K}^{2} \epsilon_{1}\left\|D\left(Y_{1}^{n+1}\right)\right\|^{2}+\frac{d^{\prime} K^{2}}{4 \epsilon_{1}}\left\|Y_{1}^{n+1}\right\|^{2}, \\
v\left|\left(U_{1}^{n+1}, U_{1}^{n, u}\right)\right| \leqslant\left\|U_{1}^{n+1}\right\|^{2}+\frac{v^{2}}{4}\left\|U_{1}^{n, u}\right\|^{2}, \\
\left|\lambda_{1} b\left(\mathbf{u}_{1}^{h, n}, U_{1}^{n+1}, U_{1}^{n+1}\right)\right| \leqslant \frac{\lambda_{1}}{2}\left(d^{\prime}\left\|\nabla Y_{1}^{n}\right\|_{\infty}\left\|U_{1}^{n+1}\right\|^{2}+M\left\|U_{1}^{n+1}\right\|^{2}\right), \\
2 \alpha v\left|\left(D\left(Y_{1}^{n+1}\right), U_{1}^{n, u}\right)\right| \leqslant 4 \alpha^{2} \epsilon_{2}\left\|D\left(Y_{1}^{n+1}\right)\right\|^{2}+\frac{v^{2}}{4 \epsilon_{2}}\left\|U_{1}^{n, u}\right\|^{2} .
\end{gathered}
$$


Now, we estimate each term of $\mathbf{F}\left(Y_{1}^{n+1}, U_{1}^{n+1}\right)$. As $\mathbf{F}$ has many terms and in order to make the proof more clear, we divide the terms of $\mathbf{F}$ into four parts, that is, $c(\cdot, \cdot, \cdot)$ terms of $\mathbf{F}, b(\cdot, \cdot, \cdot)$ terms of $\mathbf{F}, g_{a}$ terms of $\mathbf{F}$, other terms of $\mathbf{F}$. Firstly, we estimate $c(\cdot, \cdot, \cdot)$ terms of $\mathbf{F}$;

$$
\begin{aligned}
2 \alpha \operatorname{Re}\left|c\left(\mathbf{u}_{1}^{h, n}, \Phi_{1}^{n+1}, Y_{1}^{n+1}\right)\right| \leqslant 2 \alpha( & \left.\operatorname{Re}^{2}\left\|Y_{1}^{n+1}\right\|^{2}+\frac{K^{2} d^{\prime}}{4}\left\|\nabla \Phi_{1}^{n+1}\right\|^{2}\right) . \\
2 \alpha \operatorname{Re}\left|c\left(\mathbf{u}_{1}^{h, n}-\mathbf{u}^{n+1}, \mathbf{u}^{n+1}, Y_{1}^{n+1}\right)\right| \leqslant 2 \alpha( & \frac{\operatorname{Re}^{2} d^{\prime 2} M^{2}}{2}\left\|Y_{1}^{n}\right\|^{2}+\frac{3}{2} \alpha\left\|Y_{1}^{n+1}\right\|^{2} \\
& \left.+\frac{\operatorname{Re}^{2} d^{\prime 2} M^{2}}{2}\left\|\Phi_{1}^{n}\right\|^{2}+\frac{\operatorname{Re}^{2}}{2} d^{\prime 2} M^{2} k \int_{t^{n}}^{t^{n+1}}\left\|\mathbf{u}_{t}\right\|^{2} d t\right) .
\end{aligned}
$$

For the $b(\cdot, \cdot, \cdot)$ terms of $\mathbf{F}\left(Y_{1}^{n+1}, U_{1}^{n+1}\right)$, we have

$$
\begin{aligned}
\lambda_{1}\left|b\left(u_{1}^{h, n}, \Psi_{1}^{n+1}, \bar{U}_{1}^{n+1}\right)\right| \leqslant & \lambda_{1}^{2}\left\|U_{1}^{n+1}\right\|^{2}+v^{2}\left\|U_{1}^{n, u}\right\|^{2}+\frac{K^{2} d^{\prime}}{2}\left\|\nabla \Psi_{1}^{n+1}\right\|^{2} \\
\lambda_{1}\left|\left(b\left(\mathbf{u}_{1}^{h, n}-\mathbf{u}^{n+1}, \sigma^{n+1}, \bar{U}_{1}^{n+1}\right)\right)\right| \leqslant & \lambda_{1}^{2}\left\|U_{1}^{n+1}\right\|^{2}+\lambda_{1}^{2} v^{2}\left\|U_{1}^{n, u}\right\|^{2}+1.5 d^{\prime 3} M^{2}\left\|Y_{1}^{n}\right\|^{2} \\
& +1.5 d^{\prime 3} M^{2}\left\|\Phi_{1}^{n}\right\|^{2}+1.5 d^{\prime 3} M^{2} k \int_{t^{n}}^{t^{n+1}}\|\mathbf{u}\|^{2} d t \\
\left|\left(\lambda-\lambda_{1}\right) b\left(\mathbf{u}^{n+1}, \sigma^{n+1}, \bar{U}_{1}^{n+1}\right)\right| \leqslant & \frac{1}{2}\left(\lambda-\lambda_{1}\right)^{2} M^{4}+\left\|U_{1}^{n+1}\right\|^{2}+v^{2}\left\|U_{1}^{n, u}\right\|^{2} .
\end{aligned}
$$

For the $g_{a}$ terms of $\mathbf{F}\left(Y_{1}^{n+1}, U_{1}^{n+1}\right)$, we can obtain

$$
\begin{aligned}
& \lambda\left|g_{a}\left(\sigma_{1}^{h, n}, \nabla \mathbf{u}_{1}^{h, n}\right)-g_{a}\left(\sigma^{n+1}, \nabla \mathbf{u}^{n+1}\right), \bar{U}_{1}^{n+1}\right| \\
& \leqslant \frac{8 d^{\prime 2} K^{2} \lambda^{2}}{\epsilon_{6}}\left\|U_{1}^{n+1}\right\|^{2}+\epsilon_{3}\left\|\nabla Y_{1}^{n}\right\|^{2}+\frac{8 d^{\prime 2} K^{2} \lambda^{2}}{\epsilon_{3}} v^{2}\left\|U_{1}^{n, u}\right\|^{2} \\
&+8 d^{\prime 2} K^{2}\left\|\nabla \Phi_{1}^{n}\right\|^{2}+5 \lambda^{2}\left\|U_{1}^{n+1}\right\|^{2}+5 \lambda^{2} v^{2}\left\|F_{1}^{n, u}\right\|^{2} \\
&+8 d^{\prime 2} K^{2} \lambda^{2} k \int_{t^{n}}^{t^{n+1}}\left\|\nabla \mathbf{u}_{t}\right\|^{2} d t+8 d^{\prime 2} M^{2}\left\|U_{1}^{n}\right\|^{2} \\
&+8 d^{\prime 2} M^{2}\left\|\Psi_{1}^{n}\right\|^{2}+8 d^{\prime 2} M^{2} k \int_{t^{n}}^{t^{n+1}}\left\|\sigma_{t}\right\|^{2} d t .
\end{aligned}
$$


Now, we estimate other terms of $\mathbf{F}\left(Y_{1}^{n+1}, U_{1}^{n+1}\right)$;

$$
\begin{aligned}
& 2 \alpha\left|\left(p^{n+1}, \nabla \cdot Y_{1}^{n+1}\right)\right| \leqslant 2 \alpha\left(C_{K}^{2} \epsilon_{4}\left\|D\left(Y_{1}^{n+1}\right)\right\|^{2}+\frac{d^{\prime}}{4 \epsilon_{4}}\left\|p^{n+1}-\widetilde{p}^{n+1}\right\|^{2}\right), \\
& 2 \alpha \operatorname{Re}\left|\left(d_{t} \Phi_{1}^{n+1}, Y_{1}^{n+1}\right)\right| \leqslant 2 \alpha\left(\operatorname{Re}^{2}\left\|Y_{1}^{n+1}\right\|^{2}+\frac{1}{4}\left\|d_{t} \Phi_{1}^{n+1}\right\|^{2}\right) \\
& 4 \alpha(1-\alpha)\left|D\left(\Phi_{1}^{n+1}\right), D\left(Y_{1}^{n+1}\right)\right| \leqslant 2 \alpha(1-\alpha)\left(\epsilon_{5}\left\|D\left(Y_{1}^{n+1}\right)\right\|^{2}+\frac{\left\|D\left(\Phi_{1}^{n+1}\right)\right\|^{2}}{\epsilon_{5}}\right), \\
& 2 \alpha\left|\left(\Psi_{1}^{n+1}, D\left(Y_{1}^{n+1}\right)\right)\right| \leqslant 2 \alpha\left(\epsilon_{6}\left\|D\left(Y_{1}^{n+1}\right)\right\|^{2}+\frac{1}{4 \epsilon_{6}}\left\|\Psi_{1}^{n+1}\right\|^{2}\right) \\
& 2 \alpha \operatorname{Re}\left(d_{t} \mathbf{u}^{n+1}-\mathbf{u}_{t}^{n+1}, Y_{1}^{n+1}\right) \leqslant 2 \alpha\left(\operatorname{Re}^{2}\left\|Y_{1}^{n+1}\right\|^{2}+\frac{1}{4}\left\|d_{t} \mathbf{u}^{n+1}-\mathbf{u}_{t}^{n+1}\right\|^{2}\right) \\
& \lambda\left|\left(d_{t} \Psi_{1}^{n+1}, U_{1}^{n+1}\right)\right| \leqslant \lambda^{2}\left\|U_{1}^{n+1}\right\|^{2}+\frac{1}{4}\left\|d_{t} \Psi_{1}^{n+1}\right\|^{2}, \\
& 2 \alpha\left|\left(D\left(\Phi_{1}^{n+1}\right), \bar{U}_{1}^{n+1}\right)\right| \leqslant\left\|U_{1}^{n+1}\right\|^{2}+v^{2}\left\|U_{1}^{n, u}\right\|^{2}+2 \alpha^{2}\left\|\nabla \Phi_{1}^{n+1}\right\|^{2}, \\
&\left|\Psi_{1}^{n+1}, \bar{U}_{1}^{n+1}\right| \leqslant\left\|U_{1}^{n+1}\right\|^{2}+v^{2}\left\|U_{1}^{n, u}\right\|^{2}+\frac{1}{2}\left\|\Psi_{1}^{n+1}\right\|^{2}, \\
& \lambda\left|\left(d_{t} \sigma^{n+1}-\sigma_{t}^{n+1}, U_{1}^{n+1}\right)\right| \leqslant \lambda^{2}\left\|U_{1}^{n+1}\right\|^{2}+\frac{1}{4}\left\|d_{t} \sigma^{n+1}-\sigma_{t}^{n+1}\right\|^{2}, \\
&\left|\left(\lambda \sigma_{t}^{n+1}, v U_{1}^{n, u}\right)\right| \leqslant \lambda^{2}\left(2+d^{\prime}\left\|\nabla Y_{1}^{n}\right\| \infty\right)\left\|U_{1}^{n+1}\right\| \|^{2} \\
&+\frac{v^{2}}{4}\left(d^{\prime 2} M^{2}+d^{\prime}\left\|\nabla Y_{1}^{n}\right\|_{\infty}\right)\left\|\sigma_{t}^{n+1}\right\|^{2}+\frac{v^{2}}{4} K^{2} d^{\prime}\left\|\nabla \sigma_{t}^{n+1}\right\|^{2} .
\end{aligned}
$$

Combining the estimates in (5.18)-(5.21), we obtain the estimate for $\mathbf{F}\left(Y_{1}^{n+1}, U_{1}^{n+1}\right)$ :

$$
\begin{aligned}
\mathbf{F}\left(Y_{1}^{n+1}, U_{1}^{n+1}\right) \leqslant & 2 \alpha\left(C_{K}^{2} \epsilon_{4}+(1-\alpha) \epsilon_{5}+\epsilon_{6}\right)\left\|D\left(Y_{1}^{n+1}\right)\right\|^{2}+C_{K}^{2} \epsilon_{3}\left\|D\left(Y_{1}^{n+1}\right)\right\|^{2} \\
& +2 \alpha\left(3 \operatorname{Re}^{2}+1.5\right)\left\|Y_{1}^{n+1}\right\|^{2}+\left(\frac{3 d^{\prime} M^{2}}{2}+\alpha \operatorname{Re}^{2} d^{\prime 2} M^{2}\right)\left\|Y_{1}^{n}\right\|^{2} \\
& +\left\{d^{\prime}\left\|\nabla Y_{1}^{n}\right\|_{\infty}+3+9 \lambda^{2}+2 \lambda_{1}^{2}+\frac{8 d^{\prime 2} K^{2} \lambda^{2}}{\epsilon_{3}}\right\}\left\|U_{1}^{n+1}\right\|^{2}+8 d^{\prime 2} M^{2}\left\|U_{1}^{n}\right\|^{2} \\
& +v^{2}\left(5 \lambda^{2}+\lambda_{1}^{2}+4+\frac{8 d^{\prime 2} K^{2} \lambda^{2}}{\epsilon_{3}}\right)\left\|U_{1}^{n, u}\right\|^{2}+\frac{2 \alpha d^{\prime}}{4 \epsilon_{4}}\left\|p^{n+1}-\tilde{p}^{n+1}\right\|^{2} \\
& +\left(\alpha \operatorname{Re}^{2} d^{\prime 2} M^{2}+\frac{3 d^{\prime 3} M^{2}}{2}\right)\left\|\Phi_{1}^{n}\right\|^{2}+2 \alpha\left(\frac{K^{2} d^{\prime}}{4}+\frac{1-\alpha}{\epsilon_{5}}+2 \alpha^{2}\right)\left\|\nabla \Phi_{1}^{n+1}\right\|^{2}
\end{aligned}
$$


Discrete Dynamics in Nature and Society

$$
\begin{aligned}
& +8 d^{\prime 2} K^{2}\left\|\nabla \Phi_{1}^{n}\right\|^{2}+\frac{\alpha}{2}\left\|d_{t} \Phi_{1}^{n+1}\right\|^{2}+\frac{1}{4}\left\|d_{t} \Psi_{1}^{n+1}\right\|^{2}+\left(\frac{\alpha}{2 \epsilon_{6}}+\frac{1}{2}\right)\left\|\Psi_{1}^{n+1}\right\|^{2} \\
& +\frac{d^{\prime} K^{2}}{2}\left\|\nabla \Psi_{1}^{n+1}\right\|^{2}+8 d^{\prime 2} M^{2}\left\|\Psi_{1}^{n}\right\|^{2}+\frac{\alpha}{2}\left\|d_{t} \mathbf{u}^{n+1}-\mathbf{u}_{t}^{n+1}\right\|^{2}+\frac{1}{4}\left\|d_{t} \sigma^{n+1}-\sigma_{t}^{n+1}\right\|^{2} \\
& +\frac{v^{2}}{4}\left(d^{\prime}\left\|\nabla Y_{1}^{n}\right\|_{\infty}+d^{\prime 2} M^{2}\right) \cdot\left\|\sigma_{t}^{n+1}\right\|^{2}+\left(\alpha \operatorname{Re}^{2} d^{\prime 2} M^{2} k+1.5 d^{\prime 3} M^{2} k\right) \\
& \times \int_{t^{n}}^{t^{n+1}}\left\|\mathbf{u}_{t}\right\|^{2} d t+\frac{v^{2}}{4} K^{2} d^{\prime}\left\|\nabla \sigma_{t}^{n+1}\right\|^{2}+8 d^{\prime 2} M^{2} k \int_{t^{n}}^{t^{n+1}}\left\|\sigma_{t}\right\|^{2} d t \\
& +8 d^{\prime 2} K^{2} k \int_{t^{n}}^{t^{n+1}}\left\|\nabla \mathbf{u}_{t}\right\|^{2} d t+\frac{1}{2}\left(\lambda-\lambda_{1}\right)^{2} M^{4} .
\end{aligned}
$$

Choosing $\epsilon_{1}=\left(1-\alpha / 12 C_{K}^{2} \operatorname{Re}^{3} \alpha\right), \epsilon_{2}=(1-\alpha / 12 \alpha), \epsilon_{3}=\left(2 \alpha(1-\alpha) / 6 C_{K}^{2}\right), \epsilon_{4}=(1-$ $\left.\alpha / 6 C_{K}^{2}\right), \epsilon_{5}=1 / 6, \epsilon_{6}=(1-\alpha) / 6$, and substituting (5.17) and (5.22) into (5.16) yields

$$
\begin{aligned}
\alpha \operatorname{Re} \| & Y_{1}^{s}\left\|^{2}+\frac{\lambda}{2}\right\| U_{1}^{s}\left\|^{2}+2 \alpha(1-\alpha) \sum_{n=0}^{s-1} k\right\| D\left(Y_{1}^{n+1}\right)\left\|^{2}+k \sum_{n=0}^{s-1}\right\| U_{1}^{n+1} \|^{2} \\
& +\left(v \lambda_{1}-v^{2}\left(\frac{7 \alpha}{2(1-\alpha)}+\frac{28 d^{\prime 2} C_{K}^{2} K^{2} \lambda^{2}}{\alpha(1-\alpha)}+\frac{17}{4}+5 \lambda^{2}+\lambda_{1}^{2}\right)\right) k \sum_{n=0}^{s-1}\left\|U_{1}^{n, u}\right\|^{2} \\
\leqslant & C_{3} k \sum_{n=0}^{s-1}\left\|Y_{1}^{n+1}\right\|^{2}+k \sum_{n=0}^{s-1}\left(C_{4}\left(1+\left\|\nabla Y_{1}^{n}\right\|_{\infty}\right)\right)\left\|U_{1}^{n+1}\right\|^{2}+C_{5} k \sum_{n=0}^{s-1}\left\|\Phi_{1}^{n+1}\right\|^{2} \\
& +C_{6} k \sum_{n=0}^{s-1}\left\|\nabla \Phi_{1}^{n+1}\right\|^{2}+\frac{\alpha}{2} k \sum_{n=0}^{s-1}\left\|d_{t} \Phi_{1}^{n+1}\right\|^{2}+\frac{1}{4} k \sum_{n=0}^{s-1}\left\|d_{t} \Psi_{1}^{n+1}\right\|^{2} \\
& +C_{7} k \sum_{n=0}^{s-1}\left\|\Psi_{1}^{n+1}\right\|^{2}+C_{8} k \sum_{n=0}^{s-1}\left\|\nabla \Psi_{1}^{n+1}\right\|^{2}+\frac{\alpha}{2} k \sum_{n=0}^{s-1}\left\|d_{t} u^{n+1}-u_{t}^{n+1}\right\|^{2} \\
& +\frac{1}{4} k \sum_{n=0}^{s-1}\left\|d_{t} \sigma^{n+1}-\sigma_{t}^{n+1}\right\|^{2}+\frac{3 \alpha d^{\prime} C_{K}^{2}}{1-\alpha} k \sum_{n=0}^{s-1}\left\|p^{n+1}-\tilde{p}^{n+1}\right\|^{2} \\
& +k \sum_{n=0}^{s-1} \frac{v}{4}\left(d^{\prime 2} M^{2}+d^{\prime}\left\|\nabla Y_{1}^{n}\right\| \|_{\infty}\right)\left\|\sigma_{t}^{n+1}\right\|^{2}+k^{2}\left(\alpha \operatorname{Re}{ }^{2} d^{\prime 2} M^{2}+1.5 d^{\prime 3} M^{2}\right)\left\|u_{t}\right\|_{0,0}^{2} \\
& +K^{2} d^{\prime} \frac{v^{2}}{4}\left\|\sigma_{t}\right\|_{0,1}^{2}+8 d^{\prime 2} k^{2}\left(M^{2}\left\|\sigma_{t}\right\|_{0,0}^{2}+K^{2}\left\|u_{t}\right\|_{0,1}^{2}\right)+k \sum_{n=0}^{s-1} \frac{1}{2}\left(\lambda-\lambda_{1}\right)^{2} M^{4} .
\end{aligned}
$$


According to the approximating properties and the definition (3.5), we can get

$$
\begin{aligned}
& k \sum_{n=0}^{s-1}\left\|\nabla \Phi_{1}^{n+1}\right\|^{2}+k \sum_{n=0}^{s-1}\left\|\nabla \Psi_{1}^{n+1}\right\|^{2} \leqslant C\left(k \sum_{n=0}^{s-1}\left(h^{4}\left\|u^{n+1}\right\|_{3}^{2}+h^{2}\left\|\sigma^{n+1}\right\|_{2}^{2}\right)\right) \\
& \leqslant C\left(h^{4}\left|\|u\|_{0,3}^{2}+h^{2}\right|\|\sigma\|_{0,2}^{2}\right) \text {, } \\
& k \sum_{n=0}^{s-1}\left\|\Phi_{1}^{n+1}\right\|^{2}+k \sum_{n=0}^{s-1}\left\|\Psi_{1}^{n+1}\right\|^{2}+k \sum_{n=0}^{s-1}\left\|p^{n+1}-\tilde{p}^{n+1}\right\|^{2} \leqslant C\left(h^{6} k \sum_{n=0}^{s-1}\left\|u^{n+1}\right\|_{3}^{2}+h^{4} k \sum_{n=0}^{s-1}\left\|\sigma^{n+1}\right\|_{2}^{2}\right. \\
& \left.+h^{4} k \sum_{n=0}^{s-1}\left\|p^{n+1}\right\|_{2}^{2}\right) \\
& \leqslant C\left(h^{6}|\|u\||_{0,3}^{2}+\left.h^{4}\left|\|\sigma\|_{0,2}^{2}+h^{4}\right|\|p\|\right|_{0,2} ^{2}\right) \text {, } \\
& k \sum_{n=0}^{s-1}\left\|d_{t} \Phi_{1}^{n+1}\right\|^{2} \leqslant C h^{6}\left\|u_{t}\right\|_{0,3}^{2} \\
& k \sum_{n=0}^{s-1}\left\|d_{t} \Psi_{1}^{n+1}\right\|^{2} \leqslant C h^{4} \mid\left\|u_{t}\right\|_{0,2}^{2} \\
& k \sum_{n=0}^{s-1}\left\|d_{t} u^{n+1}-u_{t}^{n+1}\right\|^{2} \leqslant \frac{1}{3} k^{2}\left\|u_{t t}\right\|_{0,0}^{2} \\
& k \sum_{n=0}^{s-1}\left\|d_{t} \sigma^{n+1}-\sigma_{t}^{n+1}\right\|^{2} \leqslant \frac{1}{3} k^{2}\left\|\sigma_{t t}\right\|_{0,0}^{2} .
\end{aligned}
$$

Combining the inequalities (5.24)-(5.29) with (5.23), we yield

$$
\begin{aligned}
& \alpha \operatorname{Re}\left\|Y_{1}^{s}\right\|^{2}+\frac{\lambda}{2}\left\|U_{1}^{s}\right\|^{2}+k \sum_{n=0}^{s-1}\left(2 \alpha(1-\alpha)\left\|D\left(Y_{1}^{n+1}\right)\right\|^{2}+\frac{v}{2} \lambda_{1}\left\|U_{1}^{n, u}\right\|^{2}\right) \\
& \leqslant C k \sum_{n=0}^{s-1}\left(\left\|Y_{1}^{n+1}\right\|^{2}+\left\|U_{1}^{n+1}\right\|^{2}+\left\|\nabla Y_{1}^{n}\right\|_{\infty}\left\|U_{1}^{n+1}\right\|^{2}\right)+C v^{2}\left|\left\|\sigma_{t}\right\|\right|_{0,1}^{2} \\
&+\frac{v^{2}}{4} k \sum_{n=0}^{s-1}\left\|\nabla Y_{1}^{n}\right\|_{\infty}\left\|\sigma_{t}^{n+1}\right\|^{2}+C k^{2}\left(\left\|u_{t}\right\|_{0,1}^{2}+\left\|\sigma_{t}\right\|_{0,0}^{2}+\left\|u_{t t}\right\|_{0,0}^{2}+\left\|\sigma_{t t}\right\|_{0,0}^{2}\right) \\
&+C h^{6}\left|\|u\|_{0,3}^{2}+C h^{4}\right|\|\sigma\|_{0,2}^{2}+C h^{4}|\|p\||_{0,2}^{2}+C h^{4}\left|\|u\|_{0,3}^{2}+C h^{6}\right|\left\|u_{t}\right\| \|_{0,3}^{2} \\
&+C h^{2}\left|\|\sigma\|_{0,2}^{2}+C h^{4}\right|\left\|\sigma_{t}\right\|_{0,2}^{2}+\frac{1}{2}\left(\lambda-\lambda_{1}\right)^{2} T M^{4},
\end{aligned}
$$


with $v$ chosen such that

$$
v \leqslant \frac{\lambda_{1}}{2}\left(\frac{7 \alpha}{2(1-\alpha)}+\frac{28 d^{\prime 2} C_{K}^{2} K^{2} \lambda^{2}}{\alpha(1-\alpha)}+\frac{17}{4}+5 \lambda^{2}+\lambda_{1}^{2}\right)^{-1}
$$

Applying the induction hypothesis (IH2) and the discrete Gronwall's lemma [29] to (5.30), we have

$$
\left\|Y_{1}^{s}\right\|^{2}+\left\|U_{1}^{s}\right\|^{2} \leqslant H(k, h, v)
$$

where

$$
\begin{aligned}
H(k, h, v)= & C\left(\left.h^{4}\left|\|u\|_{0,3}^{2}+h^{6}\right|\left\|u_{t}\right\|\right|_{0,3} ^{2}+\left.h^{2}\left|\|\sigma\|_{0,2}^{2}+h^{4}\right|\left\|\sigma_{t}\right\|\right|_{0,2} ^{2}+h^{4}|\|p\||_{0,2}^{2}\right) \\
& +C k^{2}\left(\left\|u_{t}\right\|_{0,1}^{2}+\left\|u_{t t}\right\|_{0,0}^{2}+\left\|\sigma_{t}\right\|_{0,1}^{2}+\left\|\sigma_{t t}\right\|_{0,0}^{2}\right) \\
& +C v^{2}\left(\mid\left\|\sigma_{t}\right\|\left\|_{0,1}^{2}+\right\|\left\|\sigma_{t}\right\| \|_{\infty, 0}^{2}\right)+C\left(\lambda-\lambda_{1}\right)^{2} T .
\end{aligned}
$$

That is to say, under the induction hypotheses (IH1) and (IH2), we establish that the inequality (5.32) is right for all $1 \leqslant s \leqslant n$, and consequently for all $n: 0 \leqslant n \leqslant T / k$, the inequality (5.32) is right. In the next subsection, we will prove that the induction hypotheses (IH1) and (IH2) are right.

Let us continue to prove the inequality (5.1).

Using triangle inequality, the estimate (5.32), approximation properties (5.5), and (5.24)-(5.25), we have

$$
\begin{aligned}
\left|\left\|\mathbf{u}-\mathbf{u}_{1}^{h}\right\|\right|_{\infty, 0}^{2}+\left|\left\|\sigma-\sigma_{1}^{h}\right\|\right|_{\infty, 0}^{2} & \leqslant\left|\left\|Y_{1}\right\|\right|_{\infty, 0}^{2}+\left|\left\|\Phi_{1}\right\|\right|_{\infty, 0}^{2}+\left|\left\|U_{1}\right\|\right|_{\infty, 0}^{2}+\left|\left\|\Psi_{1}\right\|\right|_{\infty, 0}^{2} \\
& \leqslant H(k, h, v)+C\left(h^{6}\left|\|u\|_{\infty, 3}^{2}+h^{4}\right|\|\sigma\| \|_{\infty, 2}^{2}\right) .
\end{aligned}
$$

Similarly, by means of triangle inequality, the estimate (5.32) and (5.30), approximation properties (5.5) and (5.24)-(5.25), we can obtain

$$
\begin{aligned}
\left|\left\|\mathbf{u}-\mathbf{u}_{1}^{h}\right\|\right|_{0,1}^{2}+\left|\left\|\sigma-\sigma_{1}^{h}\right\|\right|_{0,0}^{2} & \leqslant\left|\left\|Y_{1}\right\|\right|_{0,1}^{2}+\left|\left\|\Phi_{1}\right\|\right|_{0,1}^{2}+\left|\left\|U_{1}\right\|\right|_{0,0}^{2}+\left|\left\|\Psi_{1}\right\|\right|_{0,0}^{2} \\
& \leqslant C(T)\left(H(k, h, v)+h^{6}|\|\mathbf{u}\||_{0,3}^{2}+h^{4} \mid\|\sigma\| \|_{0,2}^{2}\right) .
\end{aligned}
$$

Thus, we complete the proof of Substep 1.1. That is the inequality (5.1) is right if the induction hypotheses (IH1) and (IH2) are right.

Substep 1.2. In this subsection, we will prove that the two induction hypotheses (IH1) and (IH2) for $j=1$ are right. We first verify the induction hypothesis (IH1). 
Assume (IH1) is right for any $n=0,1, \ldots, s-1$, we will prove that (IH1) is right for $n=s$. By approximating properties (5.5), inverse inequality [26], and (5.32), we have

$$
\begin{aligned}
\left\|u_{1}^{h, s}\right\|_{\infty} & \leqslant\left\|u_{1}^{h, s}-u^{s}\right\|_{\infty}+\left\|u^{s}\right\|_{\infty} \leqslant\left\|Y_{1}^{s}\right\|_{\infty}+\left\|\Phi_{1}^{s}\right\|_{\infty}+M \\
& \leqslant C h^{-d^{\prime} / 2}\left\|Y_{1}^{s}\right\|+C h^{-d^{\prime} / 2}\left\|\Phi_{1}^{s}\right\|+M \\
& \leqslant C\left(k h^{-d^{\prime} / 2}+v h^{-d^{\prime} / 2}+h^{\left(1-d^{\prime}\right) / 2}+h^{\left(2-d^{\prime}\right) / 2}+h^{\left(3-d^{\prime}\right) / 2}+h^{-d^{\prime} / 2}\left(\lambda-\lambda_{1}\right)\right)+M .
\end{aligned}
$$

As $C\left(k h^{-d^{\prime} / 2}+v h^{-d^{\prime} / 2}+h^{\left(1-d^{\prime}\right) / 2}+h^{\left(2-d^{\prime}\right) / 2}+h^{\left(3-d^{\prime}\right) / 2}+h^{-d^{\prime} / 2}\left(\lambda-\lambda_{1}\right)\right)$ independent of $s$, we can choose $k, v \leqslant h^{d^{\prime} / 2} / C, \lambda-\lambda_{1} \leqslant h^{d^{\prime} / 2} / C$, and get $\left\|u_{1}^{h, s+1}\right\|_{\infty} \leqslant M+6$.

We can get $\left\|\sigma_{1}^{h, s+1}\right\|_{\infty} \leqslant M+6$ by the same method. Define $K=M+6$, we confirm (IH1) is right for $n=s$.

Now we establish that the induction hypothesis (IH2) is right.

Assume (IH2) is right for any $n=0,1, \ldots, s-1$, we will prove that (IH2) is right for $n=s$. Using inverse inequality [26], Korn's inequality, and (5.30), we have

$$
\begin{aligned}
\sum_{n=0}^{s} k\left\|\nabla Y_{1}^{n}\right\|_{\infty} & =\sum_{n=0}^{s-1} k\left\|\nabla Y_{1}^{n+1}\right\|_{\infty} \quad\left(\text { as } Y_{1}^{0}=0\right) \\
& \leqslant C h^{-d^{\prime} / 2} \sum_{n=0}^{s-1} k\left\|\nabla Y_{1}^{n+1}\right\| \\
& \leqslant C h^{-d^{\prime} / 2} \sqrt{k s}\left(\sum_{n=0}^{s-1} k\left\|\nabla Y_{1}^{n+1}\right\|^{2}\right)^{1 / 2} \\
& \leqslant C \sqrt{T}\left(k h^{-d^{\prime} / 2}+v h^{-d^{\prime} / 2}+h^{\left(1-d^{\prime}\right) / 2}+h^{\left(2-d^{\prime}\right) / 2}+h^{-d^{\prime} / 2}\left(\lambda-\lambda_{1}\right)\right) \leqslant 1
\end{aligned}
$$

with the choosing $k, v, \lambda-\lambda_{1} \leqslant h^{d^{\prime} / 2} / 6 C \sqrt{T}$ and $h^{\left(2-d^{\prime}\right) / 2}, h^{\left(1-d^{\prime}\right) / 2}, h^{\left(2-d^{\prime}\right) / 2} \leqslant 1 / 6 C \sqrt{T}$.

Step 2. We will show that the inequality (5.2) is true.

In order to get the inequality (5.2), we also need induction hypotheses (IH1) and (IH2) for $j=2$. As the procedure of proof is almost same as Step 1, we only give the different places with Step 1.

Now, we combine the correction problem (4.3)-(4.4) with (5.8) and introduce the approximation error $e_{2}^{n+1}, \epsilon_{2}^{n+1}$. This gives

$$
\begin{aligned}
2 \alpha \operatorname{Re}( & \left.d_{t} e_{2}^{n+1}, \mathbf{v}\right)+\left(\lambda d_{t} \epsilon_{2}^{n+1}, \tau\right)+2 \alpha c\left(\operatorname{Re} \mathbf{u}_{2}^{h, n}, e_{2}^{n+1}, \mathbf{v}\right)+2 \alpha\left(\epsilon_{2}^{n+1}, D(\mathbf{v})\right) \\
& +4 \alpha(1-\alpha)\left(D\left(e_{2}^{n+1}\right), D(\mathbf{v})\right)+\lambda_{1} b\left(\mathbf{u}_{2}^{h, n}, \epsilon_{2}^{n+1}, \bar{\tau}\right)+\left(\epsilon_{2}^{n+1}, \bar{\tau}\right)-2 \alpha\left(D\left(e_{2}^{n+1}\right), \bar{\tau}\right) \\
= & 2 \alpha\left(p^{n+1}, \nabla \cdot \mathbf{v}\right)+2 \alpha \operatorname{Re}\left(d_{t} u^{n+1}-u_{t}^{n+1}, \mathbf{v}\right)+2 \alpha \operatorname{Re} c\left(\mathbf{u}_{2}^{h, n}-\mathbf{u}^{n+1}, u^{n+1}, \mathbf{v}\right) \\
& -\left(\lambda \sigma_{t}^{n+1}, \bar{\tau}\right)+\left(\lambda d_{t} \sigma^{n+1}, \tau\right)+\left(\lambda-\lambda_{1}\right) b\left(\mathbf{u}_{2}^{h, n}, \sigma_{1}^{h, n+1}-\sigma^{n+1}, \bar{\tau}\right) \\
& +\left(\lambda-\lambda_{1}\right) b\left(\mathbf{u}^{n+1}, \sigma^{n+1}, \bar{\tau}\right)+\lambda\left(g_{a}\left(\sigma_{2}^{h, n}, \nabla \mathbf{u}_{2}^{h, n}\right), \bar{\tau}\right)-\lambda\left(g_{a}\left(\sigma^{n+1}, \nabla \mathbf{u}^{n+1}\right), \bar{\tau}\right) .
\end{aligned}
$$


Substituting $e_{2}^{n+1}=Y_{2}^{n+1}+\Phi_{2}^{n+1}, \epsilon_{2}^{n+1}=U_{2}^{n+1}+\Psi_{2}^{n+1}, v=Y_{2}^{n+1}$ and $\tau=U_{2}^{n+1}$ into (5.38), we obtain

$$
\begin{aligned}
\frac{\alpha \operatorname{Re}}{k}( & \left.\left\|Y_{2}^{n+1}\right\|^{2}-\left\|Y_{2}^{n}\right\|^{2}\right)+\frac{\lambda}{2 k}\left(\left\|U_{2}^{n+1}\right\|^{2}-\left\|U_{2}^{n}\right\|^{2}\right) \\
& +4 \alpha(1-\alpha)\left(D\left(Y_{2}^{n+1}\right), D\left(Y_{2}^{n+1}\right)\right)+2 \alpha\left(U_{2}^{n+1}, D\left(Y_{2}^{n+1}\right)\right)+\left(U_{2}^{n+1}, \bar{U}_{2}^{n+1}\right) \\
& +\lambda_{1} b\left(\mathbf{u}_{2}^{h, n}, U_{2}^{n+1}, \bar{U}_{2}^{n+1}\right)-2 \alpha\left(D\left(Y_{2}^{n+1}\right), \bar{U}_{2}^{n+1}\right)+2 \alpha c\left(\operatorname{Re} \mathbf{u}_{2}^{h, n}, Y_{2}^{n+1}, Y_{2}^{n+1}\right) \\
\leqslant & 2 \alpha\left(p^{n+1}, \nabla \cdot Y_{2}^{n+1}\right)+2 \alpha \operatorname{Re}\left(d_{t} \mathbf{u}^{n+1}-\mathbf{u}_{t}^{n+1}, Y_{2}^{n+1}\right)+\left(\lambda d_{t} \sigma^{n+1}, U_{2}^{n+1}\right) \\
& +2 \alpha \operatorname{Re} c\left(\mathbf{u}_{2}^{h, n}-\mathbf{u}^{n+1}, \mathbf{u}^{n+1}, Y_{2}^{n+1}\right)-\lambda_{1} b\left(\mathbf{u}_{2}^{h, n}, \Psi_{2}^{n+1}, \bar{U}_{2}^{n+1}\right) \\
& -\left(\lambda \sigma_{t}^{n+1}, \bar{U}_{2}^{n+1}\right)+\lambda\left(g_{a}\left(\sigma_{2}^{h, n}, \nabla \mathbf{u}_{2}^{h, n}\right), \bar{U}_{2}^{n+1}\right)-\lambda\left(g_{a}\left(\sigma^{n+1}, \nabla \mathbf{u}^{n+1}\right), \bar{U}_{2}^{n+1}\right) \\
& -2 \alpha c\left(\operatorname{Re} \mathbf{u}_{2}^{h, n}, \Phi_{2}^{n+1}, Y_{2}^{n+1}\right)-4 \alpha(1-\alpha)\left(D\left(\Phi_{2}^{n+1}\right), D\left(Y_{2}^{n+1}\right)\right) \\
& -2 \alpha\left(\Psi_{2}^{n+1}, D\left(Y_{2}^{n+1}\right)\right)-\left(\lambda-\lambda_{1}\right) b\left(\mathbf{u}_{2}^{h, n}, \Psi_{1}^{n+1}, \bar{U}_{2}^{n+1}\right) \\
& +\left(\lambda-\lambda_{1}\right) b\left(\mathbf{u}^{n+1}, \sigma^{n+1}, \bar{U}_{2}^{n+1}\right)+2 \alpha\left(D\left(\Phi_{2}^{n+1}\right), \bar{U}_{2}^{n+1}\right)-\left(\Psi_{2}^{n+1}, \bar{U}_{2}^{n+1}\right) \\
& -2 \alpha \operatorname{Re}\left(d_{t} \Phi_{2}^{n+1}, Y_{2}^{n+1}\right)-\left(\lambda d_{t} \Psi_{2}^{n+1}, U_{2}^{n+1}\right)-\left(\lambda-\lambda_{1}\right) b\left(\mathbf{u}_{2}^{h, n}, U_{1}^{n+1}, \bar{U}_{2}^{n+1}\right) .
\end{aligned}
$$

Comparing (5.39) with (5.12), we find that they are only different on the following terms:

$$
-\left(\lambda-\lambda_{1}\right) b\left(\mathbf{u}_{2}^{h, n}, \Psi_{1}^{n+1}, \bar{U}_{2}^{n+1}\right)-\left(\lambda-\lambda_{1}\right) b\left(\mathbf{u}_{2}^{h, n}, U_{1}^{n+1}, \bar{U}_{2}^{n+1}\right) .
$$

So we will only deal with these terms. We have

$$
\begin{aligned}
\left|b\left(\mathbf{u}_{2}^{h, n}, \Psi_{1}^{n+1}, \bar{U}_{2}^{n+1}\right)\right| & \leqslant \frac{d^{\prime} k^{2}}{2}\left\|\nabla \Psi_{1}^{n+1}\right\|^{2}+\left\|U_{2}^{n+1}\right\|^{2}+v^{2}\left\|U_{2}^{n, \mathrm{u}}\right\|^{2} \\
& \leqslant \frac{d^{\prime} k^{2}}{2} c h^{2}\left\|\sigma^{n+1}\right\|_{2}^{2}+\left\|U_{2}^{n+1}\right\|^{2}+v^{2}\left\|U_{2}^{n, \mathrm{u}}\right\|^{2}, \\
\left|b\left(\mathbf{u}_{2}^{h, n}, \mathrm{U}_{1}^{n+1}, \bar{U}_{2}^{n+1}\right)\right| & \leqslant \frac{d^{\prime} k^{2}}{2}\left\|\nabla U_{1}^{n+1}\right\|^{2}+\left\|U_{2}^{n+1}\right\|^{2}+v^{2}\left\|U_{2}^{n, \mathrm{u}}\right\|^{2} \\
& \leqslant \frac{d^{\prime} k^{2} c h^{-2}}{2}+\left\|U_{1}^{n+1}\right\|^{2}+\left\|U_{2}^{n+1}\right\|^{2}+v^{2}\left\|U_{2}^{n, \mathrm{u}}\right\|^{2},
\end{aligned}
$$

To conclude, repeat the proof of the first statement of Theorem 5.1, replacing $\mathbf{u}_{1}^{h, n}, \sigma_{1}^{h, n}$, $Y_{1}^{n}, \Phi_{1}^{n}, U_{1}^{n}$ and $\Psi_{1}^{n}$ by $\mathbf{u}_{2}^{h, n}, \sigma_{2}^{h, n}, Y_{2}^{n}, \Phi_{2}^{n}, U_{2}^{n}$, and $\Psi_{2}^{n}$, respectively, using Korn inequality, 
Table 1: Defect step approximation for $\lambda=5.0$.

\begin{tabular}{ccccccccc}
\hline $1 / h$ & $\left|\left\|\epsilon_{1}\right\|\right|_{\infty, 0}$ & $r$ & $\left|\left\|e_{1}\right\|\right|_{\infty, 0}$ & $r$ & $\left|\left\|\epsilon_{1}\right\|\right|_{0,0}$ & $r$ & $\left.\left\|e_{1}\right\|\right|_{0,1}$ & $r$ \\
\hline 4 & 0.06643 & - & 0.003406 & - & 0.02316 & - & 0.01945 & - \\
8 & 0.01957 & 1.76 & 0.001178 & 1.53 & 0.006350 & 1.87 & 0.004860 & 2.00 \\
16 & 0.005087 & 1.94 & $3.181 e-4$ & 1.89 & 0.001598 & 1.99 & 0.001174 & 2.05 \\
32 & 0.001469 & 1.79 & $8.135 e-5$ & 1.97 & $4.268 e-4$ & 1.90 & $2.937 e-4$ & 2.00 \\
\hline
\end{tabular}

Table 2: Correction step approximation for $\lambda=5.0$.

\begin{tabular}{ccccccccc}
\hline $1 / h$ & $\left|\left\|\epsilon_{2}\right\|\right|_{\infty, 0}$ & $r$ & $\left|\left\|e_{2}\right\|\right|_{\infty, 0}$ & $r$ & $\left|\left\|\epsilon_{2}\right\|\right|_{0,0}$ & $r$ & $\left\|\left|e_{2} \|\right|_{0,1}\right.$ & $r$ \\
\hline 4 & 0.06643 & - & 0.003410 & - & 0.02316 & - & 0.01945 & - \\
8 & 0.01957 & 1.76 & 0.001180 & 1.53 & 0.006347 & 1.87 & 0.004861 & 2.00 \\
16 & 0.005087 & 1.94 & $3.191 e-4$ & 1.89 & 0.001590 & 2.00 & 0.001173 & 2.05 \\
32 & 0.001284 & 1.99 & $8.151 e-5$ & 1.97 & $3.995 e-4$ & 1.99 & $2.917 e-4$ & 2.01 \\
\hline
\end{tabular}

approximation properties (5.5), (5.24)-(5.29), and the bound $\left\|Y_{1}^{n+1}\right\|^{2}+\left\|U_{1}^{n+1}\right\|^{2} \leqslant H(k, h, v)$. Hence, we can obtain

$$
\left|\left\|\mathbf{u}-\mathbf{u}_{2}^{h}\right\|\right|_{\infty, 0}+||\left|\sigma-\sigma_{2}^{h}\left\|\left.\right|_{\infty, 0}+\left|\left\|\mathbf{u}-\mathbf{u}_{2}^{h}\right\|\right|_{0,1}+\left|\left\|\sigma-\sigma_{2}^{h}\right\|\right|_{0,0} \leqslant B(k, h, v) .\right.\right.
$$

\section{Numerical Results}

In this section, numerical results for the defect correction method applied to viscoelastic fluid flow are presented using two test problems. The first example is a known analytical solution to verify numerical convergence rates for the defect correction method. The second example simulates viscoelastic flow through a four-to-one contraction flow, a prototypical problem for viscoelastic fluid flow. As mentioned above, continuous piecewise quadratic elements were used for modeling the velocity, and continuous piecewise linear elements were used for the pressure and stress. The constitutive equation was stabilized using an SUPG discretization with parameter $v$. In this paper, we will not investigate the influence of the parameter $v$, thus, we set $v=0.6 \mathrm{~h}$.

The defect correction algorithms are implemented using public domain finite element software [30]. Linear systems are solved using the UMFPACK solver. We use the stopping criterion defined by $\max \left\{\left\|\mathbf{u}_{i}^{h}-\mathbf{u}_{i-1}^{h}\right\|,\left\|\sigma_{i}^{h}-\sigma_{i-1}^{h}\right\|\right\} \leqslant 10^{-8}$ for the iterative solver in both the defect step and the correction step of the method. We also set the maximum number of iteration equal to 15 .

Example 6.1 (Analytical solution). The theoretical convergence rates were verified by considering fluid flow across a unit square with a known solution. As in $[7,31], \Omega=[0,1]^{2}$, 
Table 3: Defect step approximation for $\mathcal{\lambda}=1000$.

\begin{tabular}{ccccccccc}
\hline $1 / h$ & $\left|\left\|\epsilon_{1}\right\|\right|_{\infty, 0}$ & $r$ & $\mid\left\|e_{1}\right\|_{\infty, 0}$ & $r$ & $\left|\left\|\epsilon_{1}\right\|\right|_{0,0}$ & $r$ & $\left.\left\|e_{1}\right\|\right|_{0,1}$ & $r$ \\
\hline 4 & 0.06710 & - & 0.003523 & - & 0.02352 & - & 0.01959 & - \\
8 & 0.01962 & 1.77 & 0.001210 & 1.54 & 0.006419 & 1.87 & 0.004885 & 2.00 \\
16 & 0.005090 & 1.95 & $3.255 e-4$ & 1.89 & 0.001613 & 1.99 & 0.001178 & 2.05 \\
32 & 0.001493 & 1.77 & $8.319 e-5$ & 1.97 & $4.306 e-4$ & 1.91 & $2.949 e-4$ & 1.99 \\
\hline
\end{tabular}

Table 4: Correction step approximation for $\lambda=1000$.

\begin{tabular}{ccccccccc}
\hline $1 / h$ & $\left|\left\|\epsilon_{2}\right\|\right|_{\infty, 0}$ & $r$ & $\left|\left\|e_{2}\right\|\right|_{\infty, 0}$ & $r$ & $\left.\left\|\epsilon_{2}\right\|\right|_{0,0}$ & $r$ & $\left.\left\|e_{2}\right\|\right|_{0,1}$ & $r$ \\
\hline 4 & 0.06710 & - & 0.003526 & - & 0.02352 & - & 0.01959 & - \\
8 & 0.01962 & 1.77 & 0.001213 & 1.54 & 0.006416 & 1.87 & 0.004886 & 2.00 \\
16 & 0.005090 & 1.95 & $3.266 e-4$ & 1.89 & 0.001605 & 2.00 & 0.001178 & 2.05 \\
32 & 0.001284 & 1.99 & $8.336 e-5$ & 1.97 & $4.030 e-4$ & 1.99 & $2.927 e-4$ & 2.01 \\
\hline
\end{tabular}

and chosen functions are added to the right-hand sides of (2.1) such that the true solution to the problem is given by

$$
\begin{gathered}
u=\left(\begin{array}{c}
10 x^{2}(x-1)^{2} y(y-1)(2 y-1) e^{-t} \\
-10 x(x-1)(2 x-1) y^{2}(y-1)^{2} e^{-t}
\end{array}\right) \\
p=10(2 x-1)(2 y-1) e^{-t} \\
\sigma=2 \alpha D(\mathbf{u})
\end{gathered}
$$

Let $r$ be the experimental global rate of convergence given by $r=\log \left(E r / E r^{\prime}\right) / \log \left(h / h^{\prime}\right)$, where $h$ and $h^{\prime}$ denote two consecutive mesh sizes with corresponding global errors $E r$ and $E r^{\prime}$. In this example, we select $\operatorname{Re}=1, a=0, \alpha=0.5$. The numerical results for Example 6.1 are presented in Tables 1, 2, 3, and 4 .

To reduce the influence of the time discretization error, the time step is taken to be very small: $k=O\left(h^{2}\right)$.

For $\lambda=5, \lambda=1000$ and the final time $T=0.1$, the calculated convergence rates in Tables 1-4 confirm what is predicted by Theorem 5.1 for continuous $\left(P_{2}, P_{1}, P_{1}\right)$ elements in space. In fact, our numerical convergence rates are better than the theoretical ones. We will find the reason in future work.

Example 6.2 (Four-to-one contraction flow). Numerical simulations of viscoelastic flow through a planar or axisymmetric contraction have been widely studied [32, 33]. Here the case of planar flow through a contraction geometry with a ratio of $4: 1$ with respect to upstream and downstream channel widths is considered. The contraction angle is a fixed $3 \pi / 2$ and the channel lengths are sufficiently long to impose a fully developed Poiseuille flow in the inflow and outflow channels. The geometry of the computational domain is illustrated in Figure 1. The lower left corner of the domain corresponds to $x=y=0$. The computations of the mesh are also shown in Figure 1 with $\Delta x_{\min }=0.0625$ and $\Delta y_{\min }=0.015625$. We denote $\Gamma_{\text {in }}=\{(x, y): x=0,0 \leqslant y \leqslant 1\}$ and $\Gamma_{\text {out }}=\{(x, y): x=8,0 \leqslant y \leqslant 0.25\}$. On this domain the velocity boundary conditions are $u_{1}=(1 / 32)\left(1-y^{2}\right), u_{2}=0$, on $\Gamma_{\text {in }}$ 


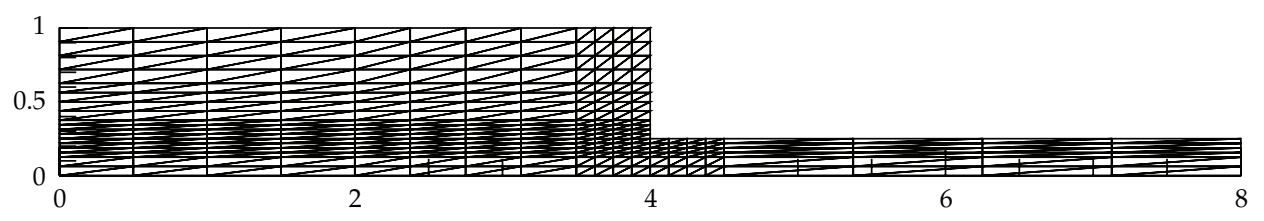

Figure 1: Plot of 4 : 1 contraction domain geometry and sample mesh.

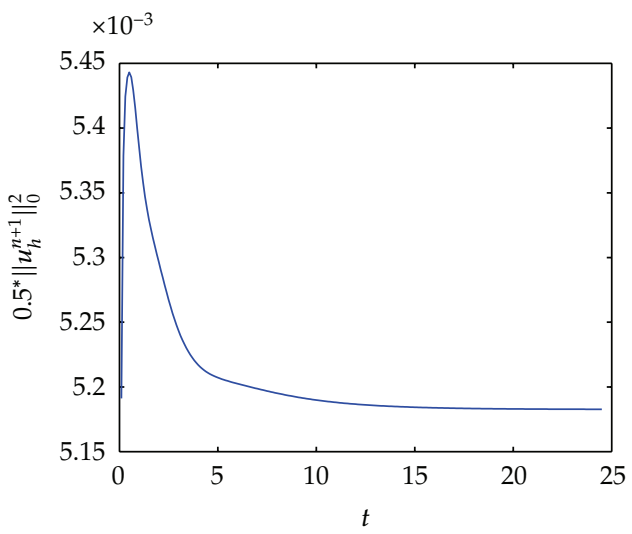

(a)

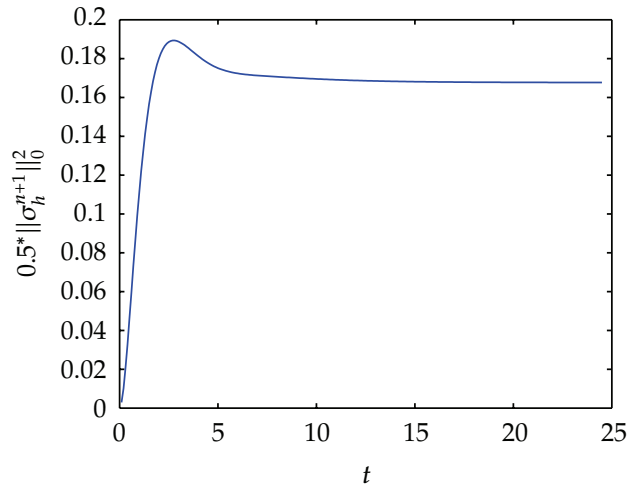

(b)

Figure 2: Evolution of $\left\|\mathbf{u}_{h}^{n+1}\right\|_{0}^{2} / 2$ (a) and $\left\|\sigma_{h}^{n+1}\right\|_{0}^{2} / 2$ (b) in time with time step $k=0.1$.

and $u_{1}=2\left((1 / 16)-y^{2}\right), u_{2}=0$, on $\Gamma_{\text {out }}$. On $\Gamma_{\text {in }}$, specified boundary conditions for $\sigma$ are given as follows: $\sigma_{11}=\left(-\alpha \lambda(a+1)(-y / 16)^{2}\right) /\left(\left(a^{2}-1\right) \lambda^{2}(-y / 16)^{2}-1\right), \sigma_{12}=\sigma_{21}=$ $-\alpha(-y / 16) /\left(\left(a^{2}-1\right) \lambda^{2}(-y / 16)^{2}-1\right), \sigma_{22}=-\alpha \lambda(a-1)(-y / 16)^{2} /\left(\left(a^{2}-1\right) \lambda^{2}(-y / 16)^{2}-1\right)$. Symmetry conditions are imposed on the bottom of the computational domain. In this example, the parameters $\operatorname{Re}, \alpha, \lambda$, and $a$ are set to $1,8 / 9,1.3$, and 0 , respectively.

We performed the following study: starting from rest, we measured the time that the approximation solution reaches a steady state. The criterion to stop this process is the following:

$$
\max \left\{\frac{\left\|\mathbf{u}_{h}^{n+1}-\mathbf{u}_{h}^{n}\right\|_{L^{2}(\Omega)}}{\left\|\mathbf{u}_{h}^{n+1}\right\|_{L^{2}(\Omega)}}, \frac{\left\|\sigma_{h}^{n+1}-\sigma_{h}^{n}\right\|_{L^{2}(\Omega)}}{\left\|\sigma_{h}^{n+1}\right\|_{L^{2}(\Omega)}}\right\} \leqslant 10^{-5},
$$

where $n+1, n$ denote $t_{n+1}, t_{n}$, respectively.

In Figure 2, we plot the evolution of the kinetic energy $\left\|\mathbf{u}_{h}^{n+1}\right\|_{0}^{2} / 2$ and $\left\|\sigma_{h}^{n+1}\right\|_{0}^{2} / 2$ using time step $k=0.1$ until it reaches its steady state, where we observe they convergence towards a steady state and also the absence of oscillations along the process.

Figure 3 presents the horizontal and vertical velocities near the reentrance corner along the vertical line $x=4.0625$ for $\lambda=1.3$. We observe that the horizontal velocity is almost continuous, while the vertical velocity has high gradients near $y=0.11$ and $y=0.23$ from Figure 3. However, we find that the solutions of the time-dependent problem can converge to the solutions of the steady problem. Figure 4 present the streamlines of the fluid with $\lambda=1.3$. 


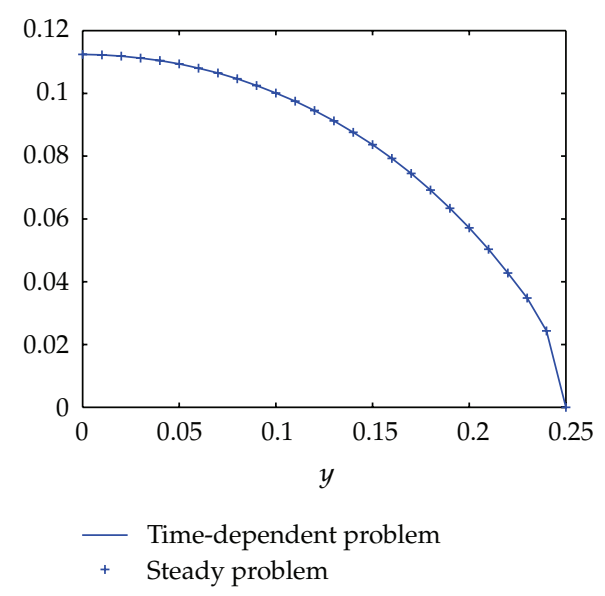

(a)

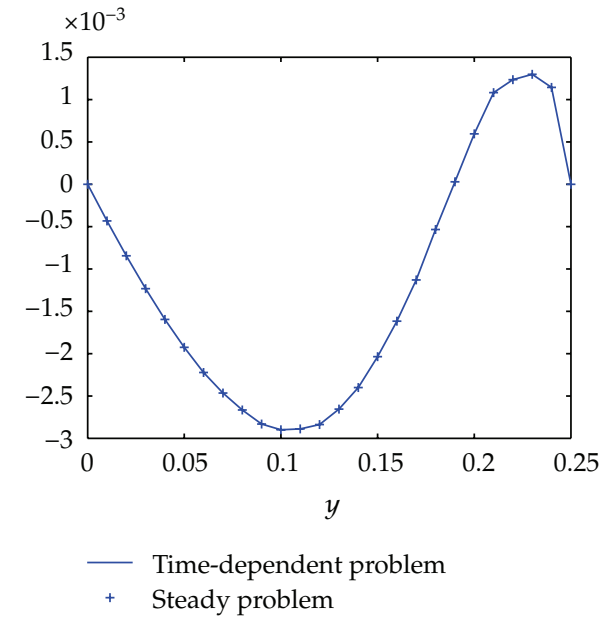

(b)

Figure 3: Horizontal (a) and vertical (b) velocity near reentrant corner. The marks " + " indicate results for steady problem and "-" indicate results for the time-dependent problem at time $t=24.5$ using time step $k=0.1$.

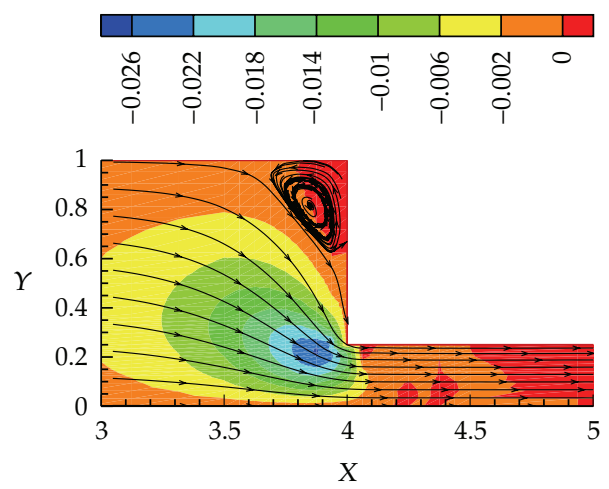

(a)
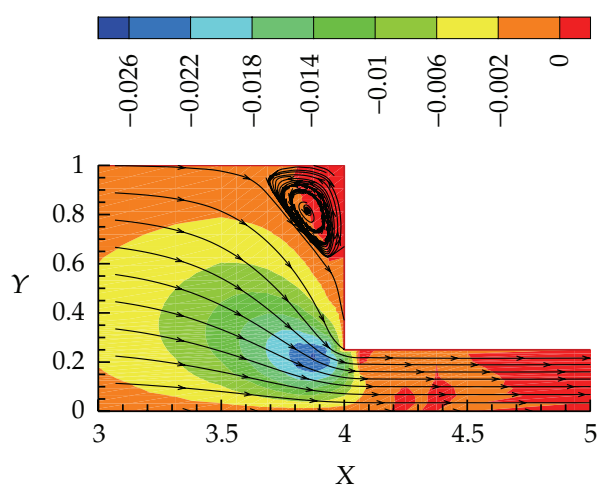

(b)

Figure 4: Streamlines and magnitude of velocity contours for $\mathbf{u}$. (a): steady problem, (b): time-dependent problem at time $t=24.5$ using $k=0.1$.

We plot the streamlines for both the steady problem and the time-dependent problem at final time $t=24.5$. It is easy to observe that these two figures are almost alike.

\section{Conclusions}

In this paper, we present a defect correction mixed finite element method for solving the timedependent Johnson-Segalman viscoelastic equations. A priori error estimates for the defect step and the first correction step of the defect correction method are derived. Finally, we present two numerical examples. One is a known problem and the other is a benchmark problem. 
For simplicity, in this paper, we choose the parameter $v=0.6 \mathrm{~h}$. More appropriate choice of parameter $v$ is currently under investigation. Further developments should be extending the method to other non-Newtonian flow or other discrete scheme.

\section{Acknowledgments}

The authors would like to thank the editor and the anonymous referees for their criticism and valuable comments, which led to the improvement of this paper. The authors want to thank Professor J. S. Howell for meaty the discussion of part program code. This paper was supported by the National Natural Science Foundation of China (no. 10871156, 10861014) and the Fund of $\mathrm{Xi}^{\prime}$ an Jiaotong University (no. 2009xjtujc30).

\section{References}

[1] J. Baranger and S. Wardi, "Numerical analysis of a FEM for a transient viscoelastic flow," Computer Methods in Applied Mechanics and Engineering, vol. 125, no. 1-4, pp. 171-185, 1995.

[2] V. J. Ervin and W. W. Miles, "Approximation of time-dependent viscoelastic fluid flow: SUPG approximation," SIAM Journal on Numerical Analysis, vol. 41, no. 2, pp. 457-486, 2003.

[3] V. J. Ervin and W. W. Miles, "Approximation of time-dependent, multi-component, viscoelastic fluid flow," Computer Methods in Applied Mechanics and Engineering, vol. 194, no. 18-20, pp. 2229-2255, 2005.

[4] T. J. Hughes and A. N. Brooks, A multidimensional Upwind Scheme with No Crosswind Diffusion, in Finite Element Methods for Convection Dominated Flows, New York, NY, USA, 1979.

[5] M. Bensaada and D. Esselaoui, "Error estimates for a stabilized finite element method for the Oldroyd B model," Journal of Mathematical Analysis and Applications, vol. 325, no. 2, pp. 1042-1059, 2007.

[6] V. J. Ervin and N. Heuer, "Approximation of time-dependent, viscoelastic fluid flow: Crank-Nicolson, finite element approximation," Numerical Methods for Partial Differential Equations, vol. 20, no. 2, pp. 248-283, 2004.

[7] J. C. Chrispell, V. J. Ervin, and E. W. Jenkins, "A fractional step $\theta$-method approximation of timedependent viscoelastic fluid flow," Journal of Computational and Applied Mathematics, vol. 232, no. 2, pp. 159-175, 2009.

[8] A. Bonito, P. Clément, and M. Picasso, "Mathematical and numerical analysis of a simplified timedependent viscoelastic flow," Numerische Mathematik, vol. 107, no. 2, pp. 213-255, 2007.

[9] P. Nithiarasu, "A fully explicit characteristic based split (CBS) scheme for viscoelastic flow calculations," International Journal for Numerical Methods in Engineering, vol. 60, no. 5, pp. 949-978, 2004.

[10] H. K. Lee, "Analysis of a defect correction method for viscoelastic fluid flow," Computers and Mathematics with Applications, vol. 48, no. 7-8, pp. 1213-1229, 2004.

[11] V. J. Ervin and H. Lee, "Defect correction method for viscoelastic fluid flows at high Weissenberg number," Numerical Methods for Partial Differential Equations, vol. 22, no. 1, pp. 145-164, 2006.

[12] V. J. Ervin, J. S. Howell, and H. Lee, "A two-parameter defect-correction method for computation of steady-state viscoelastic fluid flow," Applied Mathematics and Computation, vol. 196, no. 2, pp. 818-834, 2008.

[13] X. L. Luo, "An incremental difference formulation for viscoelastic flows and high resolution FEM solutions at high Weissenberg numbers," Journal of Non-Newtonian Fluid Mechanics, vol. 79, no. 1, pp. 57-75, 1998.

[14] J. Petera, "A new finite element scheme using the Lagrangian framework for simulation of viscoelastic fluid flows," Journal of Non-Newtonian Fluid Mechanics, vol. 103, no. 1, pp. 1-43, 2002.

[15] J. S. Howell, "Computation of viscoelastic fluid flows using continuation methods," Journal of Computational and Applied Mathematics, vol. 225, no. 1, pp. 187-201, 2009.

[16] K. Bhmer and H. J. Stetter, Defect Correction Methods-Theory and Applications, vol. 5 of Computing Supplementum, Springer, Vienna, Austria, 1984.

[17] R. Minero, M. J. H. Anthonissen, and R. M. M. Mattheij, "A local defect correction technique for timedependent problems," Numerical Methods for Partial Differential Equations, vol. 22, no. 1, pp. 128-144, 2006. 
[18] V. J. Ervin, W. J. Layton, and J. M. Maubach, "Adaptive defect-correction methods for viscous incompressible flow problems," SIAM Journal on Numerical Analysis, vol. 37, no. 4, pp. 1165-1185, 2000.

[19] W. Layton, H. Lee, and J. A. Peterson, "A defect-correction method for the incompressible NavierStokes equations," Applied Mathematics and Computation, vol. 129, no. 1, pp. 1-19, 2002.

[20] Q. Liu and Y. R. Hou, "A two-level defect-correction method for Navier-Stokes equations," Bulletin of the Australian Mathematical Society, vol. 81, no. 3, pp. 442-454, 2010.

[21] A. Labovschii, "A defect correction method for the time-dependent Navier-Stokes equations," Numerical Methods for Partial Differential Equations, vol. 25, no. 1, pp. 1-25, 2009.

[22] Y. Zhang, Y. Hou, and B. Mu, "Defect correction method for time-dependent viscoelastic fluid flow," International Journal of Computer Mathematics, vol. 88, no. 7, pp. 1546-1563, 2011.

[23] R. B. Bird, R.C. Armstrong, and O. Hassager, Dynamics of Polymeric Liquids, John Wiley and Sons, New York, NY, USA, 1987.

[24] C. Guillopé and J. C. Saut, "Existence results for the flow of viscoelastic fluids with a differential constitutive law," Nonlinear Analysis. Theory, Methods \& Applications, vol. 15, no. 9, pp. 849-869, 1990.

[25] M. Renardy, Mathematical Analysis of Viscoelastic Flows, vol. 73 of CBMS-NSF Regional Conference Series in Applied Mathematics, Society for Industrial and Applied Mathematics (SIAM), Philadelphia, Pa, USA, 2000.

[26] S. C. Brenner and L. R. Scott, The Mathematical Theory of Finite Element Methods, vol. 15 of Texts in Applied Mathematics, Springer, New York, NY, USA, 1994.

[27] V. Girault and P. A. Raviart, Finite Element methods for Navier-Stokes Equations, vol. 5 of Springer Series in Computational Mathematics, Springer, Berlin, Germany, 1986.

[28] P. Clément, "Approximation by finite element functions using local regularization," RAIRO Analyse Numerique, vol. 9, no. R-2, pp. 77-84, 1975.

[29] J. G. Heywood and R. Rannacher, "Finite-element approximation of the nonstationary Navier-Stokes problem. IV. Error analysis for second-order time discretization," SIAM Journal on Numerical Analysis, vol. 27, no. 2, pp. 353-384, 1990.

[30] F. Hecht, O. Pironneau, A. Le Hyaric, and K. Ohtsuka, "FreeFem++," 2009, http:/ /www.freefem.org/ $\mathrm{ff}++$.

[31] H. Lee, "A multigrid method for viscoelastic fluid flow," SIAM Journal on Numerical Analysis, vol. 42, no. 1, pp. 109-129, 2004.

[32] F. P.T. Baaijens, "Mixed finite element methods for viscoelastic flow analysis: a review," Journal of Non-Newtonian Fluid Mechanics, vol. 79, no. 2-3, pp. 361-385, 1998.

[33] R. G. Owens and T. N. Phillips, Computational Rheology, Imperial College Press, London, UK, 2002. 


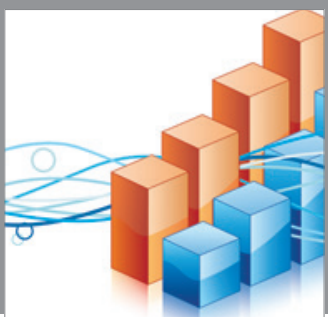

Advances in

Operations Research

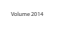

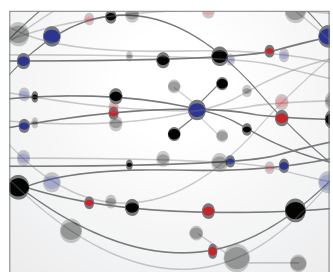

\section{The Scientific} World Journal
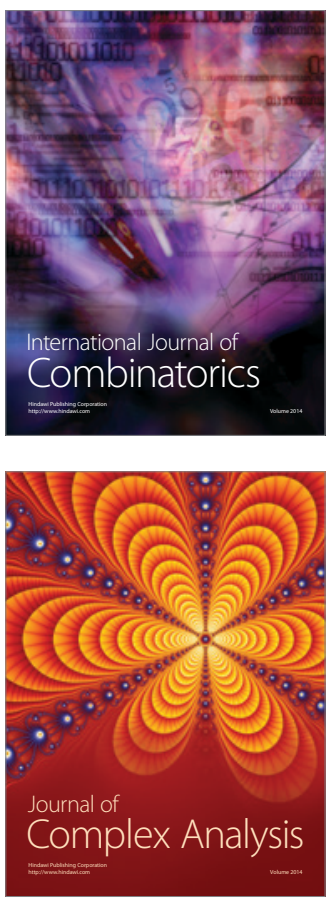

International Journal of

Mathematics and

Mathematical

Sciences
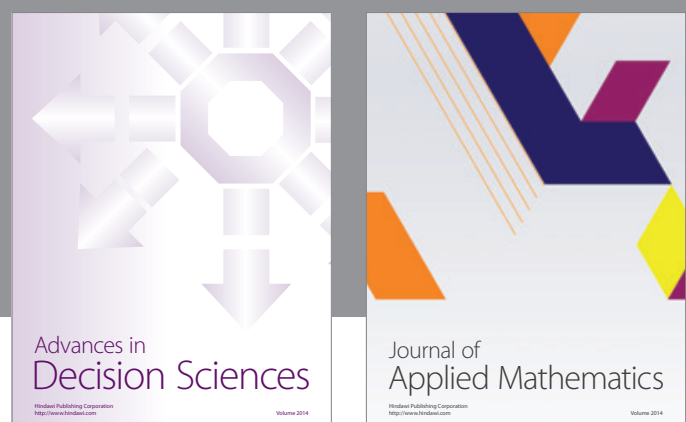

Journal of

Applied Mathematics
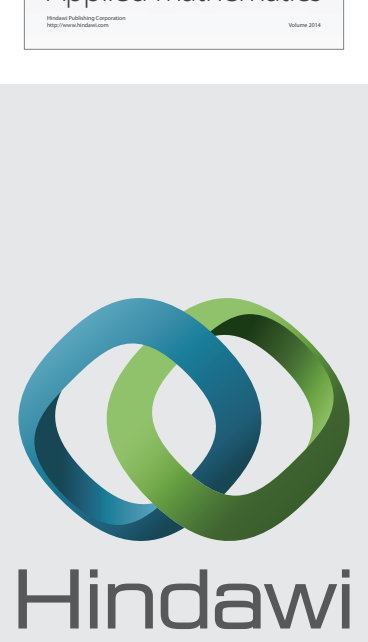

Submit your manuscripts at http://www.hindawi.com
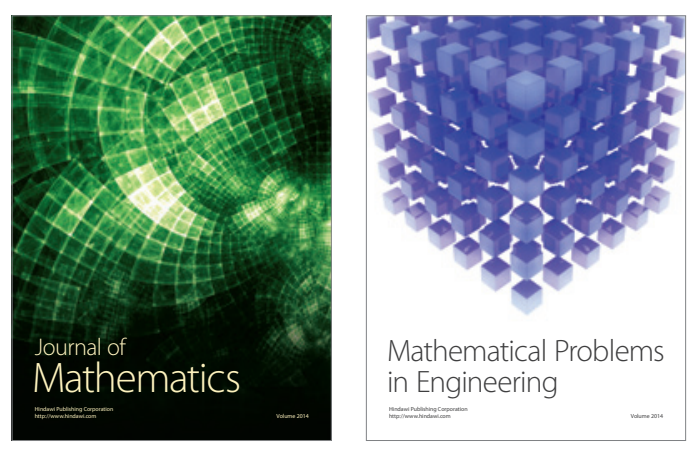

Mathematical Problems in Engineering
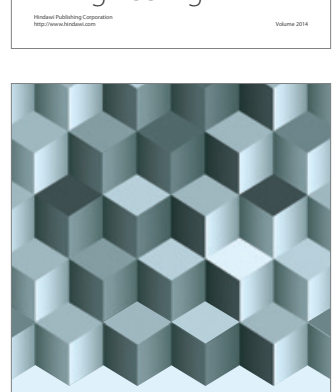

Journal of

Function Spaces
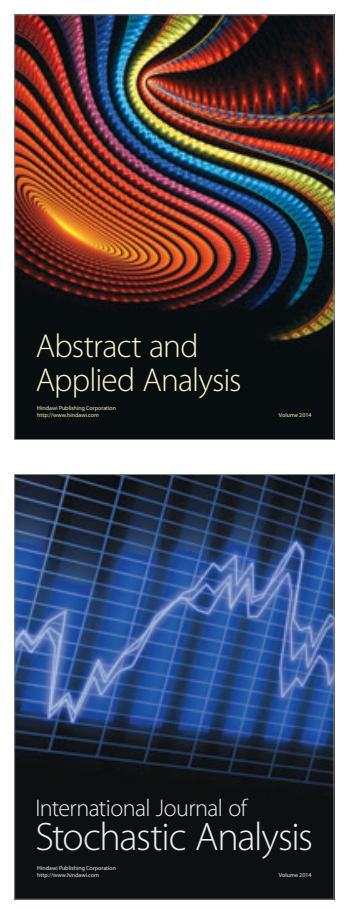

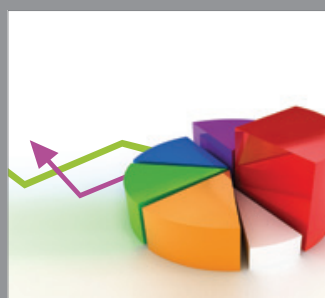

ournal of

Probability and Statistics

Promensencen
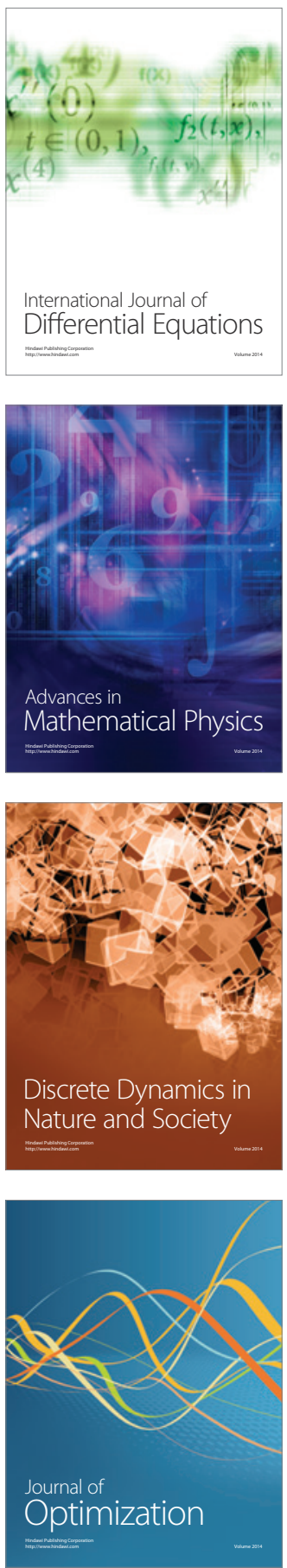